\title{
Zero-Forcing based MIMO Two-Way Relay with Relay Antenna Selection: Transmission Scheme and Diversity Analysis
}

\author{
Hui Gao, Student Member, IEEE, Tiejun Lv, Senior Member, IEEE, Shengli Zhang, \\ Chau Yuen, Senior Member, IEEE, and Shaoshi Yang, Student Member, IEEE
}

\begin{abstract}
Combining of physical-layer network coding (PNC) and multiple-input multiple-output (MIMO) can significantly improve the performance of the wireless TWRN. This paper proposes novel Max-Min optimization based relay antenna selection (RAS) schemes for zero-forcing (ZF) based MIMO-PNC transmission. RAS relaxes ZF's constraints on the number of antennas and extends the applications of ZF based MIMO-PNC to more practical scenarios, where the dedicated relay has more antennas than the end-node. Moreover, RAS also brings diversity advantages to TWRN and the achievable diversity gains of the proposed schemes are theoretically analyzed. In particular, an equivalence relation is carefully built for the diversity gains obtained by 1) RAS for ZF based MIMO-PNC and 2) transmit antenna selection (TAS) for MIMO broadcasting (BC) with ZF receivers. This equivalence transforms the original problem to a more tractable form which eventually allows explicit analytical results. It is interesting to see that Max-Min RAS keeps the network diversity gain of $\mathrm{ZF}$ based MIMO-PNC to be the same as the diversity gain of the point-to-point link within the TWRN. This insight extends the understanding on the behaviors of $\mathrm{ZF}$ transceivers with antenna selection (AS) to relatively complicated MIMO-TWRN/BC scenarios.
\end{abstract}

Index Terms-Physical-layer network coding (PNC), multipleinput multiple-output (MIMO), zero-forcing (ZF), two-way relay, antenna selection, diversity analysis.

\section{INTRODUCTION}

$\mathbf{T}$ WO-WAY relay (TWR) has been considered as a promising technique to improve spectral efficiency of the re-

Manuscript received December 26, 2011; revised June 6, 2012 and September 14, 2012; accepted September 24, 2012. T. Lv is the corresponding author. The work of H. Gao was done during his Ph.D. study at Beijing University of Posts and Telecommunications. The work of $\mathrm{H}$. Gao and T. Lv was supported in part by NSFC (Grant No. 60972075 and No. 61271188). The work of S. Zhang was supported in part by NSFC (Grant No. 20902016) and NSF Guangdong (Grant No. 10151806001000003). The work of C. Yuen was partly supported by the Singapore University Technology and Design (Grant No. SUTD-ZJU/RES/02/2011). The work of S. Yang was partly supported by China Scholarship Council. This paper was presented in part at the 2012 IEEE International Conference on Communications, Ottawa, Canada. The review of this paper was coordinated by Prof. Masoud Ardakani.

H. Gao is with Singapore University of Technology and Design, Singapore, 138682. (e-mail: hui_gao@sutd.edu.sg)

T. Lv is with the School of Information and Communication Engineering, Beijing University of Posts and Telecommunications, Beijing, 100876, China (e-mail: 1vtiejun@bupt.edu.cn).

S. Zhang is with Department of Communication Engineering, Shenzhen University, Shenzhen, 518060, China (e-mail:zsl@szu.edu.cn).

C. Yuen is with Singapore University of Technology and Design, Singapore, 138682. (e-mail: yuenchau@ @utd.edu.sg).

S. Yang is with the School of Electronics and Computer Science, University of Southampton, SO17 1BJ Southampton, U.K. (e-mail: sy7g09@ecs.soton.ac.uk). lay aided networks. In particular, the physical-layer network coding (PNC) aided TWR scheme has been proposed for the basic network of three nodes, in which two end nodes exchange information with the aid of a half-duplex relay node [1]. Unlike the traditional schemes employing three or four transmission phases for the TWR, PNC consists of only two transmission phases, namely the multiple-access (MA) phase and the broadcast $(\mathrm{BC})$ phase. Due to its great potential to improve spectral efficiency of the relay network, PNC has been attracting increasing attention since it was invented. On the other hand, multiple-input multiple-output (MIMO) technology has been successfully implemented into many advanced wireless communication systems, yielding pronounced advantages over its single-input single-output (SISO) counterparts [2], [3]. Therefore, it is highly expected that the wedding of PNC and MIMO will bring more benefits to the two-way relay network (TWRN).

Recently, many research efforts have been devoted to the combination of PNC and MIMO, where precoding techniques are generally assumed. As noted by [4], [5], if the phase of the bidirectional signals can be synchronized and the equivalent channel gains can be balanced at the relay node, the signal alignment may be accomplished, which significantly simplifies the detection and codeword mapping for decodeand-forward (DF) based SISO-PNC at the relay side. This observation is particularly useful for the spatial multiplexing oriented MIMO-PNC with DF protocol. With suitable precoding techniques, not only can the MIMO two-way relay channel (TWRC) be decoupled into multiple equivalent interferencefree SISO-TWRCs, but the signal alignment can be achieved in each SISO-TWRC as well to simplify PNC-related detection and mapping at the relay side. In this way, PNC can benefit from MIMO at a relatively low cost.

To achieve signal alignment, a zero-forcing (ZF) precoding scheme at the end-node was first proposed in [6] to facilitate the DF based MIMO-PNC transmission in MA phase, where the straightforward channel inversion and power scaling were employed. Noting that the ZF precoding scheme of [6] may not perform well under bad channel condition, a phase alignment scheme with regularized channel inversion and vector perturbation as well as power scaling optimization was proposed in [7]. Additionally, an eigen-direction alignment scheme was proposed in [8], where the optimal spatial-direction rotation and power allocation at the end-node were employed. All the schemes in [6]-[8] are featured by directly decoupling the 
MIMO-TWRC in the MA phase, making it possible to process PNC data streams in a parallel manner. Besides the channel inversion related schemes of [6]-[8], several joint-channel decomposition based schemes were proposed in [9]-[11]. To elaborate a little further, the generalized Schur Decomposition was used in [9], the combination of the generalized Singular Value Decomposition (GSVD) and QR Decomposition was used in [10], and a newly proposed Unitary Triangular Decomposition (UTD) was used in [11] with the common purpose of achieving pair-wise triangularization of the two component MIMO matrices of the MIMO-TWRC in the MA phase. Then successive interference cancellation (SIC) receiver at the relay [9], [10] or dirty-paper coding (DPC) transmitter at the end-node [11] was employed to further transform the triangular-wise equivalent channels into diagonal interferencefree channels. Consequently, signal alignment was achieved with more sophisticated mechanisms. In general, the schemes of [7]-[11] obtain power or capacity gain over the original ZF precoding based scheme of [6] at a cost of relatively higher complexity. The power scaling optimization requires iterative computation at the relay side [7]; the optimization for common rotation matrix [8] and the joint decomposition of two channel matrices [9]-[11] require global channel state information (CSI) at both the end nodes; the SIC in [9], [10] and DPC in [11] also increase system complexity. Therefore, from practical perspective, ZF based scheme [6] still deserves consideration owing to its simplicity. In addition to [6], an interesting $\mathrm{ZF}$ based transmission scheme has recently been proposed for MIMO-TWR with a practical amplify-and-forward (AF) protocol [12]. It is noted that all the aforementioned schemes in [6]-[12] only consider the special scenario where the number of antennas deployed at each end-node is no smaller than that at the relay node. This constraint, however, may hinder their applications in the practical scenario where a dedicated relay node, e.g., a base station, often has more antennas than the end nodes, e.g., the handsets of the users. It is also worth pointing out that an MSE based joint source and relay precoding design has been proposed for AF based MIMO-TWR with very flexible relay/source antenna configuration [13]. Although the scheme in [13] bears the potential to benefit from the additional antennas at the relay, this scheme focuses on the AF protocol only.

In this paper, we focus on the DF based MIMO-TWR system and extend the ZF based MIMO-PNC [6] to the more practical scenario with a dedicated $N_{R}$-antenna relay node and two $N_{T}$-antenna end nodes, where $N_{R}>N_{T} \geq 2$. To maintain the simplicity of the end-node, we propose to use $\mathrm{ZF}$ precoder and detector at the end node, the whole MIMO-PNC transmission is therefore a $\mathrm{ZF}$ based scheme. Especially, we propose to carefully select $N_{T}$ out of $N_{R}$ antennas at the relay to facilitate the bidirectional data exchange of $N_{T}$ streams. The relay antenna selection (RAS) not only relaxes the constraint on the number of antennas at the relay node [6], [12], but also provides an end-to-end diversity advantage for the ZF based MIMO-PNC transmission. Moreover, the achievable diversity order of the proposed scheme is explicitly analyzed, which extends the previous works on the point-to-point MIMO link [14], [15] to the more complicated MIMO network. To be specific, the main contributions of this paper are two folds.

Firstly, we propose Max-Min optimization based RAS approaches for $\mathrm{ZF}$ based MIMO-PNC. A general respective RAS (R-RAS) approach is first proposed, which optimizes the reliability of the two transmission phases by two independent RAS operations. In addition, a simple common RAS (CRAS) approach is proposed for system with reciprocal channel, which optimizes the overall transmission by a single RAS operation. The C-RAS can be considered as a simplified yet performance-preserving version of R-RAS under reciprocal channel condition. Although some relay/antenna selection schemes have been introduced for various two-way relay network (TWRN) with decode-and-forward (DF) protocol [16][18], they only consider single-antenna end nodes. Assuming multiple-antenna end nodes and relay node, a novel joint end/relay antenna selection scheme is proposed for TWR with practical AF protocol [19]. However, all of [16]-[19] focus on the bidirectional data exchange of single stream. In addition, [16]-[18] use all antennas/relays for signal reception in the MA phase to achieve full receive diversity, which require full radio frequency (RF) chains to simultaneously support all the antennas/relays. The proposed RAS approaches, however, are tailored for the ZF based MIMO-PNC with multiple bidirectional data-streams, which has not been suggested before. Moreover, our scheme takes the whole MIMO-PNC transmission into consideration and only uses a subset of antennas for reception and transmission in MA and BC phases respectively, which also achieves full diversity gain without requiring full RF chains to simultaneously support all the antennas. It is worth mention that the proposed RAS scheme also sheds some light on a possible approach to overcome the constraints encountered by [7]-[11].

Moreover, an unified and explicit diversity analysis is presented for ZF based MIMO-PNC with both R-RAS and CRAS. Although the transmit antenna selection (TAS) with ZF receiver [14], [15], [20], [21] as well as the ZF transceivers [22], [23] have been carefully studied for point-to-point MIMO system, no previous research has been done to analyze RAS's effects in the ZF based MIMO-TWR system with DF protocal. Note that the straightforward analysis is very difficult, we carefully build the equivalent relation between the end-to-end diversity order and the BC phase diversity order. Such equivalence transforms the intractable to the tractable. Thereafter, we extend [14], [15] to TAS in MIMO broadcasting (MIMOBC) scenario, which equivalently solves our problem. Finally, we obtain the answers on the diversity orders of 1) ZF based MIMO-PNC with R-RAS/C-RAS 2) TAS in the MIMO-BC system with arbitrary ZF receivers. Interestingly, the diversity orders in all of these systems show similar results as that of the point-to-point MIMO system. These insights extend our understanding on the behaviors of $\mathrm{ZF}$ transceivers with antenna selection (AS) in more complicated MIMO networks beyond the point-to-point MIMO link.

The rest of the paper is organized as follows. In Section II, we describe the ZF based MIMO-PNC transmission scheme with arbitrary relay antenna subset. In Section III, we obtain the Max-Min based R-RAS and R-RAS following the analysis of the performance bound with regard to the proposed scheme. 


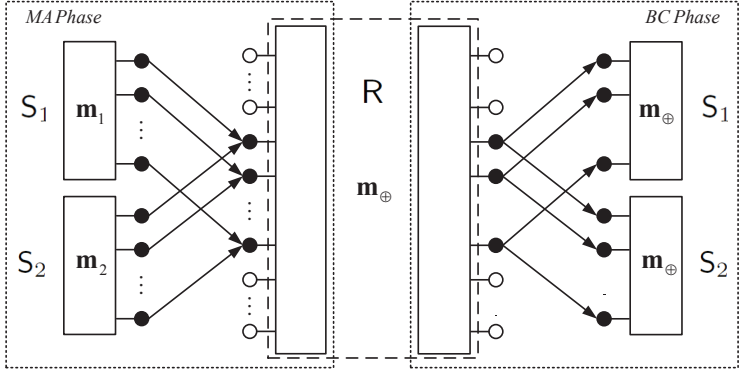

Fig. 1. ZF based MIMO-PNC with RAS, where $N_{T}$ out of $N_{R}$ relay antennas are selected in MA and BC phases to facilitate the $N_{T}$ streams of bidirectional information exchange between the end nodes. Here, $\mathbf{m}_{k}$ is the symbol vector transmitted from $\mathrm{S}_{k}, k=1,2$, and $\mathbf{m}_{\oplus}$ is the network-coded symbol vector that $R$ aims to obtain and broadcast back to $S_{1}$ and $S_{2}$.

Section IV focuses on the analysis of diversity order achieved by ZF based MIMO-PNC with both R-RAS and C-RAS. In Section $\mathrm{V}$, numerical results are given to validate the advantages of the proposed scheme and the theoretical result for diversity order. Finally, Section VI concludes this paper.

In this paper, $[\mathbf{A}]_{m, n}$ is the $(m, n)$-th entry of matrix $\mathbf{A}$; $(\cdot)^{*},(\cdot)^{T},(\cdot)^{H},(\cdot)^{-1},\|\cdot\|_{F}, \operatorname{tr}(\cdot)$ are the conjugate, transpose, conjugate transpose, inverse, Frobenius norm and trace of a matrix. $\Re(\cdot)$ takes the real part of a complex variable. $\bmod _{N}(x)$ is the modulo operation for $x$ with respect to N. $C_{n}^{m}$ is the number of $n$-combinations from a given set of $m$ elements. $\mathcal{C N}\left(\mu, \sigma^{2}\right)$ denotes a complex Gaussian variable with mean $\mu$ and variance $\sigma^{2}$.

\section{System Description}

\section{A. Notation and Assumption}

We consider a separated TWRN where two $N_{T}$-antenna $\left(N_{T} \geq 2\right)$ end nodes $\left(\mathrm{S}_{1}\right.$ and $\left.\mathrm{S}_{2}\right)$ can only exchange information via one $N_{R}$-antenna $\left(N_{R}>N_{T}\right)$ half-duplex relay node $(\mathrm{R})$, as shown in Fig. 1. We assume that the relay is located at the middle point of the line-segment determined by the two end nodes, and the large-scale fading is not considered for simplicity. The quasi-static channels between link $\mathrm{S}_{k} \rightarrow \mathrm{R}$ and $\mathrm{R} \rightarrow \mathrm{S}_{k}$ are denoted by an $N_{R} \times N_{T}$ channel matrix $\mathcal{G}_{k}$ and an $N_{T} \times N_{R}$ channel matrix $\mathcal{H}_{k}, k=1,2$. The entries in both $\mathcal{G}_{k}$ and $\mathcal{H}_{k}$ are independent and identically distributed (i.i.d.) $\mathcal{C N}(0,1)$. Let us denote $\mathbf{G}_{k}^{(\phi)}$ and $\mathbf{H}_{k}^{\left(\phi^{\prime}\right)}$ as the selected $N_{T} \times N_{T}$ channel matrices regarding the selected relay antenna subsets $\phi$ and $\phi^{\prime}$ for link $\mathrm{S}_{k} \rightarrow \mathrm{R}$ and link $\mathrm{R} \rightarrow \mathrm{S}_{k}$, respectively. Here, $\phi\left(\phi^{\prime}\right)^{1}$ is an ordered $N_{T}$-element index set containing the indices of the selected antennas at $\mathrm{R} ; \mathbf{H}_{k}^{(\phi)}$ is a sub-matrix of $\mathcal{H}_{k}$, consisting of the original column vectors of $\mathcal{H}_{k}$ with indices from $\phi$. We also enumerate the $Q=C_{N_{T}}^{N_{R}}$ possible realizations of $\phi\left(\phi^{\prime}\right)$ in an order set $\Phi=\left\{\phi_{1}, \phi_{2}, \ldots, \phi_{Q}\right\}$, where $\phi\left(\phi^{\prime}\right) \in \Phi$. It is also assumed that the relay node has global CSI for RAS, power balancing between end nodes and signal detection, and each end node has local CSI for precoding and signal detection. For the spatial multiplexing oriented MIMO-PNC transmission of multiple

\footnotetext{
${ }^{1}$ Since the results of RAS in MA phase and BC phase are not necessarily the same, we introduce both $\phi$ and $\phi^{\prime}$ here without loss of generality.
}

streams, we assume equal data-rate and uncoded transmission for all the streams. With respect to the transmitted symbol in each stream, $M$-ary PAM $(M \geq 2)$ signal is first considered in this section while the extension to $M$-ary QAM will be discussed later. We assume that both $S_{1}$ and $S_{2}$ share the same $M$-ary information symbol set $\mathcal{W}=\{0,1, \ldots, M-1\}$ and the symbol-energy normalized $M$-ary PAM constellation $\mathcal{M}=\left\{a_{M}(-M+1), a_{M}(-M+3), \ldots, a_{M}(M-1)\right\}$, where $a_{M}=\sqrt{\frac{3}{\left(M^{2}-1\right)}}$ is a symbol-power normalization factor and $|\mathcal{M}|=M$. We also define the mapping function $f_{M}: \mathcal{W} \mapsto \mathcal{M}$ as $m=f_{M}(w)=a_{M}(2 w-M+1)$, where $w \in \mathcal{W}$ and $m \in \mathcal{M}$.

With the above notations and assumptions, we proceed to describe the transmission scheme with arbitrary antenna subset pair $\left(\phi, \phi^{\prime}\right)$ in the following subsection, which will subsequently enable us to extract the relevant performance metrics for corresponding RAS schemes.

\section{B. Transmission scheme with arbitrary $\left(\phi, \phi^{\prime}\right)$}

We start the description from the transmission in the MA phase. To begin with, $\mathrm{S}_{k}$ generates the $M$-ary information vector $\mathbf{w}_{k}=\left[w_{k, 1}, w_{k, 2}, \ldots, w_{k, N_{T}}\right]^{T}$ with element $w_{k, j} \in \mathcal{W}, j=1,2, \ldots, N_{T}$, and each element is mapped to the modulated symbol in $\mathcal{M}$ as $m_{k, j}=f_{M}\left(w_{k, j}\right)=$ $a_{M}\left(2 w_{k, j}-M+1\right)$. Denoting the modulated symbol vector of $S_{k}$ as $\mathbf{m}_{k}=\left[m_{k, 1}, m_{k, 2}, \ldots, m_{k, N_{T}}\right]^{T}$, then the precoding process at $S_{k}$ is described as

$$
\mathbf{x}_{k}=\sqrt{E_{S}} \lambda_{k}^{(\phi)} \mathbf{F}_{k}^{(\phi)} \mathbf{m}_{k}, k=1,2,
$$

where $\mathbf{F}_{k}^{(\phi)}:=g_{k}^{(\phi)}\left(\mathbf{G}_{k}^{(\phi)}\right)^{-1}$ is the ZF precoder with a total power conserving factor $g_{k}^{(\phi)}:=\left\|\left(\mathbf{G}_{k}^{(\phi)}\right)^{-1}\right\|_{F}^{-1}$ ensuring $\operatorname{tr}\left(\mathbf{F}_{k}^{(\phi)}\left(\mathbf{F}_{k}^{(\phi)}\right)^{H}\right)=1, \lambda_{k}^{(\phi)}:=g_{m i n}^{(\phi)} / g_{k}^{(\phi)}$ is a network power balancing factor ${ }^{2}$ with $g_{\min }^{(\phi)}:=\min \left(g_{1}^{(\phi)}, g_{2}^{(\phi)}\right)$, and $E_{S}$ is the average transmit symbol power for $m_{k, j}$. After the precoding process, $\mathrm{S}_{k}$ radiates $\mathbf{x}_{k}$ through the $N_{T}$ transmit antennas. Note that the superscript $(\phi)$ here indicates that the channelrelated variables, such as $\lambda_{k}^{(\phi)}$ and $\mathbf{F}_{k}^{(\phi)}$ in (1), are specified by the selected relay antenna subset $\phi$ in the MA phase. With perfect synchronization, the superimposed symbols arriving at $\mathrm{R}$ are

$$
\mathbf{y}=\mathbf{G}_{1}^{(\phi)} \mathbf{x}_{1}+\mathbf{G}_{2}^{(\phi)} \mathbf{x}_{2}+\mathbf{n}
$$

where $\mathbf{y}=\left[y_{1}, y_{2}, \ldots, y_{N_{T}}\right]^{T}$ is the received signal vector, and $\mathbf{n}$ is the noise vector with elements being i.i.d. $\mathcal{C N}\left(0, N_{0}\right)$. By substituting (1) into (2), we rewrite $\mathbf{y}$ as

$$
\begin{aligned}
\mathbf{y} & =\sqrt{E_{S}} \lambda_{1}^{(\phi)} g_{1}^{(\phi)} \mathbf{m}_{1}+\sqrt{E_{S}} \lambda_{2}^{(\phi)} g_{2}^{(\phi)} \mathbf{m}_{2}+\mathbf{n} \\
& =\sqrt{E_{S}} g_{\min }^{(\phi)} \mathbf{m}_{+}+\mathbf{n}
\end{aligned}
$$

where $\mathbf{m}_{+}:=\mathbf{m}_{1}+\mathbf{m}_{2}$ is the superposition of the two signal vectors from $S_{1}$ and $S_{2}$. (3) shows that the ZF precoding decouples the MIMO-TWRC into multiple equivalent SISO-TWRCs in the MA phase, as illustrated in Fig. 1. Moreover, the network power balancing

\footnotetext{
${ }^{2}$ The power balancing factor is introduced to achieve signal alignment at the relay. With signal alignment, the detection and mapping for the PNC symbols can be simplified. Moreover, the power balancing factor also offers a mathematically tractable form for further analysis on the diversity order. In practice, the network power balancing factor can be computed by the relay and feed back to the end nodes.
} 
factors $\left\{\lambda_{1}^{(\phi)}, \lambda_{2}^{(\phi)}\right\}$ ensure that the equivalent channel amplitudes of different streams are all equal to $\sqrt{E_{S}} g_{\text {min }}^{(\phi)}$ at $\mathrm{R}$ in the MA phase. With reference to (3), the detection and mapping for PNC can be carried out in a parallel manner at R. In addition, the general nonbinary PNC mapping scheme [5] can be implemented into each of the SISO-TWRCs. Based on (3), the received superimposed signal in the $j^{\text {th }}$ equivalent SISO-TWRC, as shown in Fig. 2, is

$$
y_{j}=\sqrt{E_{S}} g_{\min }^{(\phi)} m_{+, j}+n_{j}, j=1,2, \ldots, N_{T},
$$

where $m_{+, j}:=m_{1, j}+m_{2, j} \in \mathcal{M}_{+}$is the $j^{\text {th }}$ element of $\mathbf{m}_{+}$constituting the sum-constellation of two identical $M$-ary PAM constellations $\mathcal{M}$, i.e.,

$$
\mathcal{M}_{+}=\left\{a_{M}(-2 M+2), a_{M}(-2 M+4), \ldots, a_{M}(2 M-2)\right\},
$$

and $n_{j}$ is the noise component. Based on $y_{j}$ the relay node $\mathrm{R}$ can obtain the network-coded symbol in the following two steps. Firstly, R estimates $m_{+, j}$ belonging to the $(2 M-1)$-ary PAM constellation $\mathcal{M}_{+}$with the aid of the maximum likelihood (ML) detector as

$$
\hat{m}_{+, j}=\arg \min _{\tilde{m}_{+, j} \in \mathcal{M}_{+}}\left[\Re\left(y_{j}\right)-\sqrt{E_{S}} g_{\text {min }}^{(\phi)} \tilde{m}_{+, j}\right]^{2} .
$$

Then, R employs the PNC mapping, $f_{P N C}: \mathcal{M}_{+} \mapsto \mathcal{M}$, to obtain the network-coded symbol as $m_{\oplus, j}=f_{P N C}\left(\hat{m}_{+, j}\right)=$ $a_{M} \bmod _{M}\left(\frac{1}{2 a_{M}} \hat{m}_{+, j}-1\right)$, where $\hat{m}_{+, j} \in \mathcal{M}_{+}, m_{\oplus, j} \in \mathcal{M}$. By collecting the results from the $N_{T}$ streams, the network-coded symbol vector $\mathbf{m}_{\oplus}:=\left[m_{\oplus, 1}, m_{\oplus, 2}, \ldots, m_{\oplus, N_{T}}\right]^{T}$ is obtained.

In the BC phase, $\mathrm{R}$ broadcasts the network-coded symbol vector $\mathbf{m}_{\oplus}$ back to $S_{1}$ and $S_{2}$ through the selected antennas in $\phi^{\prime}$. The received signal at $S_{k}$ is then given by

$$
\mathbf{y}_{k}=\sqrt{E_{S}} \mathbf{H}_{k}^{\left(\phi^{\prime}\right)} \mathbf{m}_{\oplus}+\mathbf{n}_{k}, k=1,2,
$$

where $\mathbf{n}_{k}$ is the noise vector with elements obeying i.i.d. $\mathcal{C N}\left(0, N_{0}\right)$. Upon receiving $\mathbf{y}_{k}$, the ZF filter is used to decouple $\mathbf{y}_{k}$ at $S_{k}$ as

$$
\mathbf{y}_{k}^{\prime}=\frac{1}{\sqrt{E_{S}}}\left(\mathbf{H}_{k}^{\left(\phi^{\prime}\right)}\right)^{-1} \mathbf{y}_{k}=\mathbf{m}_{\oplus}+\mathbf{n}_{k}^{\prime},
$$

where $\mathbf{n}_{k}^{\prime}:=\left(\mathbf{H}_{k}^{\left(\phi^{\prime}\right)}\right)^{-1} \mathbf{n}_{k}$. Similar to the operation at $\mathrm{R}$, the elements of $\mathbf{m}_{\oplus}$ is detected in a parallel manner at $S_{k}$ as

$$
\hat{m}_{\oplus, k, j}=\arg \min _{\tilde{m}_{\oplus, j} \in \mathcal{M}}\left\|\Re\left(y_{k, j}^{\prime}\right)-\tilde{m}_{\oplus, j}\right\|^{2} .
$$

With $\hat{m}_{\oplus, k, j}$ at hand, the desired information from the other end-node in the $j^{t h}$ stream is calculated with the self-interference cancellation as $\hat{w}_{3-k, j}=\bmod _{N}\left[f_{M}^{-1}\left(\hat{m}_{\oplus, k, j}\right)-w_{k, j}\right]$. Finally, by collecting the results from the $N_{T}$ streams at $\mathrm{S}_{k}$, the estimated information vector $\hat{\mathbf{w}}_{3-k}$ is obtained, and the MIMO-PNC transmission of $N_{T^{-}}$ stream is achieved.

Remark 1: The proposed scheme can be readily implemented with rectangular $M$-ary QAM. Since the proposed ZF transceivers have transformed the complex MIMO-TWRC into multiple parallel scalar SISO-TWRCs, the in-phase and the quadrature signals can be processed as two independent $\sqrt{M}$-ary PAM signals. Therefore, the following analysis will only focus on the PAM signal and the obtained results are straightforwardly applicable to the rectangular QAM signal.

\section{RElay Antenna SELECtion}

Given the channel $\mathcal{G}_{k}$ and $\mathcal{H}_{k}, k=1,2$, RAS aims to improve the overall system transmission reliability through the optimized relay antenna subset pair $\left(\phi, \phi^{\prime}\right)$. Note that the proposed ZF processing has decoupled the MIMO-TWRC into multiple equivalent SISO-TWRCs, and each SISO-TWRC consists of two equivalent SISO one-way relay

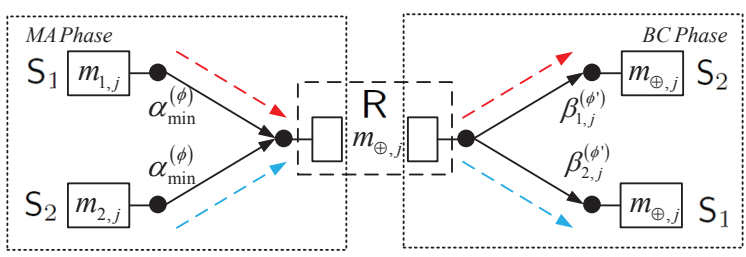

Fig. 2. The $j^{\text {th }}$ equivalent SISO-TWRC with the equivalent channel gain $\alpha_{\text {min }}^{(\phi)}$ for MA phase and $\left\{\beta_{1, j}^{\left(\phi^{\prime}\right)}, \beta_{2, j}^{\left(\phi^{\prime}\right)}\right\}$ for BC phase. Within the equivalent SISO-TWRC, the red and blue dashed arrows indicate the equivalent SISO-OWRCs for link $\mathrm{S}_{1} \rightarrow \mathrm{R} \rightarrow \mathrm{S}_{2}$ and link $\mathrm{S}_{2} \rightarrow \mathrm{R} \rightarrow \mathrm{S}_{1}$, respectively.

channels (OWRC), as shown in Fig. 2. Let us denote $P_{E 2 E, k, j}^{\left(\phi, \phi^{\prime}\right)}$ as the instantaneous (with respect to each channel fading realization) endto-end symbol-error probability (SEP) at $\mathrm{S}_{k}$ within its $j^{\text {th }}$ equivalent SISO one-way relay channel (OWRC). Following [17], [24], we then introduce a practical performance metric of the overall system as

$$
P_{E 2 E}^{\left(\phi, \phi^{\prime}\right)}=\frac{1}{2 N_{T}} \sum_{j=1}^{N_{T}} \sum_{k=1}^{2} P_{E 2 E, k, j}^{\left(\phi, \phi^{\prime}\right)},
$$

where $P_{E 2 E}^{\left(\phi, \phi^{\prime}\right)}$ is the average end-to-end SEP of the $2 N_{T}$ SISOOWRCs. In the next two sub-sections, we will first analyze $P_{E 2 E}^{\left(\phi, \phi^{\prime}\right)}$ by giving the SEP bound of each equivalent SISO-OWRC. Thereafter we will be able to extract the relevant performance metric for MaxMin optimization based RAS schemes.

\section{A. SEP Bound of the Equivalent SISO-OWRC}

Let us first focus on the $j^{\text {th }}$ equivalent SISO-OWRC $\left(\mathrm{S}_{3-k} \rightarrow\right.$ $\mathrm{R} \rightarrow \mathrm{S}_{k}$ ), and denote the SEP in MA and BC phases as $P_{M A}^{(\phi)}{ }^{4}$ and $P_{B C, k, j}^{\left(\phi^{\prime}\right)}$. Note that if a symbol detection error occurs in only one of the two phases, an end-to-end error is destined to happen at $\mathrm{S}_{k}$; on the other hand, if symbol detection errors occur in both phases, the end-to-end error happens with the probability of $P_{E 2 E \mid M \& B, k, j}^{\left(\phi, \phi^{\prime}\right)}$. By summarizing these observations, we express $P_{E 2 E, k, j}^{\left(\phi, \phi^{\prime}\right)}$ as

$$
\begin{aligned}
P_{E 2 E, k, j}^{\left(\phi, \phi^{\prime}\right)}= & P_{M A}^{(\phi)}\left(1-P_{B C, k, j}^{\left(\phi^{\prime}\right)}\right)+P_{B C, k, j}^{\left(\phi^{\prime}\right)}\left(1-P_{M A}^{(\phi)}\right) \\
& +P_{E 2 E \mid M \& B, k, j}^{\left(\phi, \phi^{\prime}\right)} P_{B C, k, j}^{\left(\phi^{\prime}\right)} P_{M A}^{(\phi)} \\
\approx & P_{M A}^{(\phi)}+P_{B C, k, j}^{\left(\phi^{\prime}\right)},
\end{aligned}
$$

which indicates that the end-to-end symbol error probability $P_{E 2 E, k, j}^{\left(\phi, \phi^{\prime}\right)}$ is approximated by the sum of the dominating terms of $P_{M A}^{(\phi)}$ and $P_{B C, k, j}^{\left(\phi^{\prime}\right)}$. We will use this approximation in the following part of this paper for analytical simplicity, and we will give the bound of $P_{E 2 E, k, j}^{\left(\phi, \phi^{\prime}\right)}$ after presenting the bounds of $P_{M A}^{(\phi)}$ and $P_{B C, k, j}^{\left(\phi^{\prime}\right)}$, respectively. To be more specific, $P_{M A}^{(\phi)}$ is first bounded as follows.

Proposition 1. Given the channel $\mathbf{G}_{k}^{(\phi)}, k=1,2$, then $P_{M A}^{(\phi)}$ is bounded by

$$
\frac{2(M-1)}{M} \mathcal{Q}_{M A}^{(\phi)} \leq P_{M A}^{(\phi)} \leq \frac{2\left(M^{2}-1\right)}{M^{2}} \mathcal{Q}_{M A}^{(\phi)},
$$

\footnotetext{
${ }^{3}$ In this paper, we omit the variables in the SEP functions for clarity, unless we need to specify them explicitly.

${ }^{4}$ After the ZF precoding and power balancing operation, the SEPs of different equivalent SISO-OWRCs are all the same in the MA phase, i.e., independent of index $k, j$ (cf.(4)).
} 
where $\mathcal{Q}_{M A}^{(\phi)}:=\mathcal{Q}\left(\sqrt{2 a_{M}^{2} \alpha_{m i n}^{(\phi)} \rho}\right), \mathcal{Q}(x)$ is the Gaussian $\mathcal{Q}$ function; $\alpha_{\text {min }}^{(\phi)}:=\left(g_{\text {min }}^{(\phi)}\right)^{2}$ can be regarded as the equivalent channel gain in the MA phase (see Fig. 2); finally, $\rho:=E_{S} / N_{0}$ is the transmit symbol signal-to-noise ratio (SNR).

Proof: According to [5], [25], $P_{M A}^{(\phi)}$ is upper bounded as,

$P_{M A}^{(\phi)} \leq \frac{\left(M^{2}-1\right)}{M^{2}} \operatorname{Pr}\left(\left|\Re\left(n_{j}\right)\right|>\frac{d_{\text {min }}}{2}\right)=\frac{2\left(M^{2}-1\right)}{M^{2}} \mathcal{Q}_{M A}^{(\phi)}$,

where $d_{\min }=2 \sqrt{E_{S}} g_{\text {min }}^{(\phi)} a_{M}$ is the minimum Euclidean distance between the constellation points in $\mathcal{M}_{+}$with the power scaling factor $\sqrt{E_{S}} g_{\text {min }}^{(\phi)}$. Similar to the approach of [24], the genie-aided detection is used for deriving the lower bound, which assumes that a genie informs $\mathrm{R}$ the information $m_{1, j}$ from $\mathrm{S}_{1}$. The detection performance of $m_{+, j}=m_{1, j}+m_{2, j}$ with known $m_{1, j}$ will be no worse than that without $m_{1, j}$. Moreover, the detection for $m_{+, j}$ with known $m_{1, j}$ degenerates to ordinary detection of $M$-PAM signal, hence the lower bound is obtained as

$$
P_{M A}^{(\phi)} \geq \frac{2(M-1)}{M} \mathcal{Q}_{M A}^{(\phi)} .
$$

Combining the upper bound and lower bound, we finish the proof.

We then proceed to analyze $P_{B C, k, j}^{\left(\phi^{\prime}\right)}$. After ZF post-processing in (7), $S_{k}$ detects the desired signals according to (8). As a result, the corresponding SEP is

$$
P_{B C, k, j}^{\left(\phi^{\prime}\right)}=\frac{2(M-1)}{M} \mathcal{Q}_{B C, k, j}^{\left(\phi^{\prime}\right)}
$$

where $\mathcal{Q}_{B C, k, j}^{\left(\phi^{\prime}\right)}:=\mathcal{Q}\left(\sqrt{2 a_{M}^{2} \beta_{k, j}^{\left(\phi^{\prime}\right)} \rho}\right), \beta_{k, j}^{\left(\phi^{\prime}\right)}:=\left\{\left[\mathbf{W}_{k}^{\left(\phi^{\prime}\right)}\right]_{j j}\right\}^{-1}$ with $\mathbf{W}_{k}^{\left(\phi^{\prime}\right)}:=\left(\left(\mathbf{H}_{k}^{\left(\phi^{\prime}\right)}\right)^{H} \mathbf{H}_{k}^{\left(\phi^{\prime}\right)}\right)^{-1}$. Here, $\beta_{k, j}^{\left(\phi^{\prime}\right)}$ is considered as the equivalent channel gain in BC phase (see Fig. 2).

Finally, by substituting the bound of $P_{M A}^{(\phi)}$ in (11) and the result of $P_{B C, k, j}^{\left(\phi^{\prime}\right)}$ in (12) into (10), the SEP bound of the equivalent SISOOWRC is expressed as

$$
\frac{2(M-1)}{M} \mathcal{Q}_{+, k, j}^{\left(\phi, \phi^{\prime}\right)} \leq P_{E 2 E, k, j}^{\left(\phi, \phi^{\prime}\right)} \leq \frac{2\left(M^{2}-1\right)}{M^{2}} \mathcal{Q}_{+, k, j}^{\left(\phi, \phi^{\prime}\right)},
$$

where $\mathcal{Q}_{+, k, j}^{\left(\phi, \phi^{\prime}\right)}:=\mathcal{Q}_{M A}^{(\phi)}+\mathcal{Q}_{B C, k, j}^{\left(\phi^{\prime}\right)}$. Note that (13) becomes increasingly tight as $M$ increases.

\section{B. Relay Antenna Selection}

With the SEP bound for SISO-OWRC, we can further quantify the SEP bound of the whole system and then extract key performance metrics to facilitate the design of RAS scheme. Specifically, by substituting (13) into (9) the bound of $P_{E 2 E}^{\left(\phi, \phi^{\prime}\right)}$ is expressed as

$$
\frac{(M-1)}{M N_{T}} \sum_{k, j} \mathcal{Q}_{+, k, j}^{\left(\phi, \phi^{\prime}\right)} \leq P_{E 2 E}^{\left(\phi, \phi^{\prime}\right)} \leq \frac{\left(M^{2}-1\right)}{M^{2} N_{T}} \sum_{k, j} \mathcal{Q}_{+, k, j}^{\left(\phi, \phi^{\prime}\right)} .
$$

Note that both the upper bound and the lower bound of $P_{E 2 E}^{\left(\phi, \phi^{\prime}\right)}$ contain the common item $\sum_{k, j} \mathcal{Q}_{+, k, j}^{\left(\phi, \phi^{\prime}\right)}$ that is relevant to $\left(\phi, \phi^{\prime}\right)$, the optimizations of $\left(\phi, \phi^{\prime}\right)$ are thus equivalent with either the upper bound or the lower bound. It is also noted that the optimizations with the explicit $P_{E 2 E}^{\left(\phi, \phi^{\prime}\right)}$ in (9) and its bounds in (14) are not equivalent in general. In this paper we mainly focus on the simple and effective suboptimal RAS schemes that are derived from the bounds in (14) or $\sum_{k, j} \mathcal{Q}_{+, k, j}^{\left(\phi, \phi^{\prime}\right)}$ with reasonable approximations.
However, the computation of the term $\sum_{k, j} \mathcal{Q}_{+, k, j}^{\left(\phi, \phi^{\prime}\right)}$ is still complicated and difficult to analyze further. Towards this end, we propose a sub-optimal RAS approach according to the following observations: 1) for the MIMO-PNC transmission of multiple streams, the overall system performance is mainly limited by the weakest SISO-OWRC with SEP bound proportional to $\mathcal{Q}_{+, \max }^{\left(\phi, \phi^{\prime}\right)}:=\mathcal{Q}_{M A}^{(\phi)}+\mathcal{Q}_{B C, \max }^{\left(\phi^{\prime}\right)}$, where $\mathcal{Q}_{B C, \text { max }}^{\left(\phi^{\prime}\right)}:=\max _{k, j}\left(\mathcal{Q}_{B C, k, j}^{\left(\phi^{\prime}\right)}\right)$, and 2) $\mathcal{Q}_{+, \text {max }}^{\left(\phi, \phi^{\prime}\right)}$ can be interpreted as the sum SEP of two links, which is also limited by the weaker one, i.e., $\max \left(\mathcal{Q}_{M A}^{(\phi)}, \mathcal{Q}_{B C, \text { max }}^{\left(\phi^{\prime}\right)}\right)$. The sub-optimal RAS is then given as

$$
\begin{aligned}
\left(\phi^{*}, \phi^{*^{\prime}}\right) & =\arg \min _{\left(\phi, \phi^{\prime}\right) \in \Phi \times \Phi}\left(\max \left(\mathcal{Q}_{M A}^{(\phi)}, \mathcal{Q}_{B C, \text { max }}^{\left(\phi^{\prime}\right)}\right)\right) \\
& =\arg \max _{\left(\phi, \phi^{\prime}\right) \in \Phi \times \Phi}\left(\min \left(\alpha_{\text {min }}^{(\phi)}, \beta_{\text {min }}^{\left(\phi^{\prime}\right)}\right)\right),
\end{aligned}
$$

where the second equation follows the monotonicity of $\mathcal{Q}$ function and $\beta_{\min }^{\left(\phi^{\prime}\right)}:=\min _{k, j}\left(\beta_{k, j}^{\left(\phi^{\prime}\right)}\right), k=1,2, j=1,2, \ldots, N_{T}$. Since the choices of $\phi$ and $\phi^{\prime}$ are independent for the independency between $\left(\alpha_{\text {min }}^{(\phi)}\right)$ and $\left(\beta_{\text {min }}^{\left(\phi^{\prime}\right)}\right)$, an optimal solution of the joint optimization for $\left(\phi, \phi^{\prime}\right)$ in (15) can be obtained by solving the following two separate optimization problems as

$$
\begin{aligned}
\mathrm{ASI}: \phi_{R, M A}^{*} & =\arg \max _{\phi \in \Phi}\left(\alpha_{m i n}^{(\phi)}\right), \\
\mathrm{AS} \mathrm{II}: \phi_{R, B C}^{*} & =\arg \max _{\phi^{\prime} \in \Phi}\left(\beta_{m i n}^{\left(\phi^{\prime}\right)}\right),
\end{aligned}
$$

where $\left(\phi_{R, M A}^{*}, \phi_{R, B C}^{*}\right)$ belongs to the optimal solution set $\left\{\left(\phi^{*}, \phi^{*^{\prime}}\right)\right\}^{5}$ of (15). Here we term the separate optimization problems of (16) and (17) as the respective relay antenna selection (R-RAS), which indicate the Max-Min optimization results $\phi_{R, M A}^{*}$ for the MA phase and $\phi_{R, B C}^{*}$ for the BC phase, regarding the equivalent channel gains in each phase. It is noted that the derivation for R-RAS does not assume channel reciprocity; therefore, R-RAS scheme can serve as a general solution for both system with/without reciprocal channel.

In fact, whenever channel reciprocity is preserved during the MA and BC phases, the implementation of R-RAS can be greatly simplified. Inspired by the reciprocal channel conditions, i.e., $\mathbf{G}_{k}^{(\phi)}=$ $\left(\mathbf{H}_{k}^{(\phi)}\right)^{T}$, we may intuitively enforce $\mathrm{R}$ to use the same antenna subset for the two-phase transmission, i.e., $\phi=\phi^{\prime}$. With reciprocal channel, the square of the power conserving factor (cf. (1)) at $S_{k}$ is rewritten as

$$
\left(g_{k}^{(\phi)}\right)^{2}=\left\|\left(\mathbf{G}_{k}^{(\phi)}\right)^{-1}\right\|_{F}^{-2}=\left\{\operatorname{tr}\left(\left(\mathbf{W}_{k}^{(\phi)}\right)^{T}\right)\right\}^{-1}
$$

where $\mathbf{W}_{k}^{(\phi)}=\left[\left(\mathbf{H}_{k}^{(\phi)}\right)^{H} \mathbf{H}_{k}^{(\phi)}\right]^{-1}$. Then an inequality is obtained to order the equivalent channel gains in MA and BC phases as

$$
\alpha_{\text {min }}^{(\phi)}=\left(g_{\text {min }}^{(\phi)}\right)^{2} \leq\left(g_{k}^{(\phi)}\right)^{2} \leq\left\{\left[\mathbf{W}_{k}^{(\phi)}\right]_{j j}\right\}^{-1}=\beta_{k, j}^{(\phi)} .
$$

By substituting $\phi=\phi^{\prime}$ into (15) and noting the inequality (19), we have the following common relay antenna selection (C-RAS) scheme as

$$
\begin{aligned}
\phi_{C}^{*} & =\arg \max _{\phi \in \Phi}\left(\min \left(\alpha_{m i n}^{(\phi)}, \beta_{m i n}^{(\phi)}\right)\right) \\
& =\arg \max _{\phi \in \Phi}\left(\alpha_{m i n}^{(\phi)}\right)=\phi_{R}^{*}, M A
\end{aligned}
$$

\footnotetext{
${ }^{5}$ The optimal solution set may contain multiple feasible elements.
} 
It is interesting to note that the C-RAS in (20) results in the same antenna subset as the AS I of the R-RAS in (16), which optimizes the MA phase transmission only. Therefore, we still refer to C-RAS in (20) as AS I without causing confusion.

Remark 2: With the ZF transceivers and power balancing operation, the resultant R-RAS and C-RAS are only determined by the channelrelated coefficients $\alpha_{\min }^{(\phi)}$ and $\beta_{m i n}^{\left(\phi^{\prime}\right)}$. Therefore, the proposed schemes are applicable with different modulation formats. In addition, C-RAS can be viewed as a tailored version of R-RAS for reciprocal channel.

\section{DIVERSITY ANALYSIS}

In this section, we aim to quantify the achievable diversity order of the proposed ZF based MIMO-PNC with R-RAS and C-RAS. In particular, we attempt to unify the diversity analysis for both RAS approaches. To accomplish this task, we first establish the equivalence between R-RAS and C-RAS in terms of diversity order, and then derive the explicit result. For analytical convenience, we introduce the exponential equality $\doteq$ as defined in [3], e.g., $f(x) \doteq x^{a}$ represents $a=-\lim _{x \rightarrow \infty} \frac{\log (f(x))}{\log (x)}$. We first focus on the R-RAS, and define the end-to-end diversity order of this scheme as

$$
\left.d_{R}:=-\lim _{\rho \rightarrow \infty}\left[\log \left(\bar{P}_{E 2 E}^{\left(\phi_{R}^{*}, M A\right.}, \phi_{R, B C}^{*}\right)(\rho)\right) / \log (\rho)\right],
$$

where $\bar{P}_{E 2 E}^{\left(\phi_{R, M A}^{*}, \phi_{R, B C}^{*}\right)}(\rho):=\mathrm{E}_{H}\left\{P_{E 2 E}^{\left(\phi_{R, M A}^{*}, \phi_{R, B C}^{*}\right)}(\rho)\right\}$ is the average overall SEP of the system (averaged over all channel realizations). Similarly, we can also define the end-to-end diversity order of C-RAS as

$$
\left.d_{C}:=-\lim _{\rho \rightarrow \infty}\left[\log \left(\bar{P}_{E 2 E}^{\left(\phi_{R}^{*}, M A\right.}, \phi_{R, M A}^{*}\right)(\rho)\right) / \log (\rho)\right],
$$

where $\bar{P}_{E 2 E}^{\left(\phi_{R, M A}^{*}, \phi_{R, M A}^{*}\right)}(\rho):=\mathrm{E}_{H}\left\{P_{E 2 E}^{\left(\phi_{R, M A}^{*}, \phi_{R, M A}^{*}\right)}(\rho)\right\}$ is the average overall SEP of the system. It is worth pointing out that, even though we have derived the SEP bound in (14), the concise approximation of $\left.\bar{P}_{E 2 E}^{\left(\phi_{R, M A}^{*}, \phi_{R, B C}^{*}\right)}\left(\bar{P}_{E 2 E}^{\left(\phi_{R}^{*}, M A\right.}, \phi_{R, M A}^{*}\right)\right)$ that illuminates $d_{R}$ $\left(d_{C}\right)$ is not easy to obtain. To circumvent this difficulty, we give the following proposition in which the diversity orders are redefined by the more tractable outage probabilities.

Proposition 2. : For ZF based MIMO-PNC transmission, the endto-end diversity orders achieved by R-RAS (with/without reciprocal channel) and C-RAS (with reciprocal channel) are the same as the diversity order of the $\mathrm{BC}$ phase achieved by R-RAS, i.e.,

$$
\left.d_{R}=d_{C}=d_{R, B C}=\lim _{x \rightarrow 0}\left[\log \left(\operatorname{Pr}\left(\beta_{\min }^{\left(\phi_{R}^{*}, B C\right.}\right)_{\leq x}\right)\right) / \log (x)\right] .
$$

Proof: The proof consists of three parts. In the first part, we redefine the diversity orders of R-RAS and C-RAS with the tractable outage probabilities. In the second part, we assume reciprocal channel and then build the equivalent relation between R-RAS and C-RAS regarding the diversity orders. Finally, we show that the diversity order obtained by R-RAS is independent of reciprocal channel. It is also noted that whenever we discuss C-RAS, the reciprocal channel condition is assumed.

Firstly, according to Lemma 1 in Appendix, $d_{R}$ can be redefined as

$$
d_{R}=\min \left(d_{R, M A}, d_{R, B C}\right)
$$

where

$$
\begin{aligned}
& \left.d_{R, M A}=\lim _{x \rightarrow 0}\left[\log \left(\operatorname{Pr}\left(\alpha_{\min }^{\left(\phi_{R}^{*}, M A\right.}\right)_{\leq x}\right)\right) / \log (x)\right], \\
& d_{R, B C}=\lim _{x \rightarrow 0}\left[\log \left(\operatorname{Pr}\left(\beta_{\min }^{\left(\phi_{R}^{*}, B C\right.} \leq x\right)\right) / \log (x)\right] .
\end{aligned}
$$

and $d_{C}$ can be redefined as

$$
\left.d_{C}=\lim _{x \rightarrow 0}\left[\log \left(\operatorname{Pr}\left(\alpha_{\min }^{\left(\phi_{R}^{*}, M A\right.}\right)_{\leq x}\right)\right) / \log (x)\right]=d_{R, M A} .
$$

It is worth mention that the definitions in (25) and (26) are independent of channel reciprocity.

After the definition of $d_{R}$ in (24) and $d_{C}$ in (27), we try to first build the equivalent relation between $d_{R}$ and $d_{C}$ under reciprocal channel. By extending the inequality in (19) with respect to matrix norm and diagonal elements, a bound of $\alpha_{\min }^{(\phi)}$ is given as

$$
\frac{1}{N_{T}} \beta_{\text {min }}^{(\phi)} \leq \alpha_{\text {min }}^{(\phi)} \leq \beta_{\text {min }}^{(\phi)}, \forall \phi \in \Phi,
$$

Then, a new bound of $\alpha_{\min }^{\left(\phi_{R, M A}^{*}\right)}$ is obtained by using (28) twice as $\left.\frac{1}{N_{T}} \beta_{\min }^{\left(\phi_{R, B C}^{*}\right)} \leq \alpha_{\min }^{\left(\phi_{R}^{*}, B C\right.} \leq \alpha_{\min }^{\left(\phi_{R, M A}^{*}\right)} \leq \beta_{\min }^{\left(\phi_{R, M A}^{*}\right)} \leq \beta_{\min }^{\left(\phi_{R}^{*}, B C\right.}\right)$

which highlights the optimality of AS I in (20) with respect to $\alpha_{\min }^{(\phi)}$ and the optimality of AS II in (17) with respect to $\beta_{m i n}^{(\phi)}$. From (29), we have

$$
\operatorname{Pr}\left(\alpha_{\min }^{\left(\phi_{R, M A}^{*}\right)} \leq x\right) \doteq \operatorname{Pr}\left(\beta_{\min }^{\left(\phi_{R, B C}^{*}\right)} \leq x\right),
$$

and consequently we have $d_{R, M A}=d_{R, B C}$. According to the equations of $d_{R}$ in (24) and $d_{C}$ in (27), we have $d_{R}=d_{C}=d_{R, B C}$ with reciprocal channel.

Let us proceed to consider the case without channel reciprocal, i.e., $\mathbf{G}_{k}^{(\phi)}$ and $\mathbf{H}_{k}^{(\phi)}$ are independent. In fact, when we study $d_{R, M A}$ here without reciprocal channel, we can assume a virtual reciprocal channel for $\mathbf{G}_{k}^{(\phi)}$ as $\tilde{\mathbf{H}}_{k}^{(\phi)}=\left(\mathbf{G}_{k}^{(\phi)}\right)^{T}$, then according to the analysis with reciprocal channel, it is easy to see that

$$
d_{R, M A}=\tilde{d}_{R, B C}=\lim _{x \rightarrow 0}\left[\log \left(\operatorname{Pr}\left(\tilde{\beta}_{m i n}^{\left(\phi_{R, B C}^{*}\right)} \leq x\right)\right) / \log (x)\right],
$$

where $\tilde{\beta}_{\min }^{\left(\phi_{R, B C}^{*}\right)}$ (defined with the victual channel $\tilde{\mathbf{H}}_{k}^{(\phi)}$ ) is the counterpart of $\beta_{\min }^{\left(\phi_{R, B C}^{*}\right)}$ (defined with the real channel $\mathbf{H}_{k}^{(\phi)}$ ). Moreover, $\tilde{\mathbf{H}}_{k}^{(\phi)}$ and $\mathbf{H}_{k}^{(\phi)}$ share the same statistical characteristics, therefore, $\tilde{d}_{R, B C}=d_{R, B C}$. Then we have $d_{R}=d_{R, B C}$ without reciprocal channel. By summarizing the above results, we finish the proof.

Based on Proposition 2, we unify the end-to-end diversity order of the ZF based MIMO-PNC with R-RAS/C-RAS as

$$
\left.d=\lim _{x \rightarrow 0}\left[\log \left(\operatorname{Pr}\left(\beta_{m i n}^{\left(\phi_{R}^{*}, B C\right.}\right) \leq x\right)\right) / \log (x)\right],
$$

then the following Proposition gives the explicitly result of $d$.

Proposition 3. For ZF based MIMO-PNC transmission, the endto-end diversity order achieved by R-RAS (with/without reciprocal channel) and C-RAS (with reciprocal channel) is $d=N_{R}-N_{T}+1$.

Proof: The proof is achieved by investigating the upper bound and lower bound respectively. Let us start from the upper bound. It is noted that the two random variables involved in $\beta_{m i n}^{(\phi)}=$ $\min \left(\beta_{1, \min }^{(\phi)}, \beta_{2, \min }^{(\phi)}\right)$ are i.i.d. due to symmetry and independence between $\mathbf{H}_{1}^{(\phi)}$ and $\mathbf{H}_{2}^{(\phi)}$. With order statistics [26], we then have

$$
\begin{aligned}
& \operatorname{Pr}\left(\beta_{\min }^{\left(\phi_{R, B C}^{*}\right)} \leq x\right) \\
= & 2 \operatorname{Pr}\left(\beta_{1, \text { min }}^{\left(\phi_{R, B C}^{*}\right)} \leq x\right)-\left[\operatorname{Pr}\left(\beta_{1, \text { min }}^{\left(\phi_{R, B C}^{*}\right)} \leq x\right)\right]^{2},
\end{aligned}
$$

By substituting (31) into (30), the upper bound of $d$ is obtained as

$$
d=d_{P 2 P}^{\left(\phi_{R, B C}^{*}\right)} \leq d_{P 2 P}^{\left(\phi_{P 2 P}^{*}\right)}=N_{R}-N_{T}+1,
$$


where $d_{P 2 P}^{\left(\phi_{R, B C}^{*}\right)}=\lim _{x \rightarrow 0}\left[\operatorname{Pr}\left(\beta_{1, \text { min }}^{\left(\phi_{R, B C}^{*}\right)} \leq x\right) / \log (x)\right]$, and the last inequation in (32) follows Lemma 2 in Appendix which emphasizes the optimality of $\phi_{P 2 P}^{*}$ in the MIMO point-to-point link.

For the lower bound, we first show that

$$
\begin{aligned}
\operatorname{Pr}\left(\beta_{\min }^{\left(\phi_{R, B C}^{*}\right)} \leq x\right) & =\operatorname{Pr}\left(\beta_{\text {min }}^{\left(\phi_{1}\right)} \leq x, \beta_{\text {min }}^{\left(\phi_{2}\right)} \leq x, \ldots, \beta_{\text {min }}^{\left(\phi_{Q}\right)} \leq x\right) \\
& =\operatorname{Pr}\left(\bigcup_{i=1}^{N_{u}} \mathrm{~A}_{i}\right)
\end{aligned}
$$

where the $N_{u}=\left(2 N_{T}\right)^{Q}$ sub-events $\left\{A_{i}\right\}$ are defined as

$$
A_{i}=\left\{\beta_{u_{i}(1), l_{i}(1)}^{\left(\phi_{1}\right)} \leq x, \beta_{u_{i}(2), l_{i}(2)}^{\left(\phi_{2}\right)} \leq x, \ldots, \beta_{u_{i}(Q), l_{i}(Q)}^{\left(\phi_{Q}\right)} \leq x\right\}
$$

for $i=1,2, \ldots, N_{u}$, and $u_{i}(q)$ is a pick from the end node indices $\{1,2\}, l_{i}(q)$ is a pick from the relay antenna index set $\phi_{q}, q=$ $1,2, \ldots, Q$. Let us define the set containing the random variables in $A_{i}$ as $S_{i}$ and according Lemma 3 in Appendix, there exists such a subset as

$$
S_{i n d, i}=\left\{\beta_{k_{1}, j_{1}}^{\left(\phi_{1}^{\prime}\right)}, \beta_{k_{2}, j_{2}}^{\left(\phi_{2}^{\prime}\right)}, \ldots, \beta_{k_{N_{R}-N_{T}+1}, j_{N_{R}-N_{T}+1}}^{\left(\phi_{N_{R}-N_{2}}^{\prime}\right.}\right\} \subset S_{i}, \forall i
$$

where $\left\{\beta_{k_{m}, j_{m}}^{\left(\phi_{m}^{\prime}\right)}\right\}_{m=1}^{N_{R}-N_{T}+1}$ are i.i.d. random variables. Recall that the equivalent channel gain $\beta_{k, j}^{(\phi)}$ is defined as $\beta_{k, j}^{(\phi)}=$ $\left\{\left[\mathbf{W}_{k}^{(\phi)}\right]_{j j}\right\}^{-1}$ wherein $\mathbf{W}_{k}^{(\phi)}=\left(\left(\mathbf{H}_{k}^{(\phi)}\right)^{H} \mathbf{H}_{k}^{(\phi)}\right)^{-1}$ and $\mathbf{H}_{k}^{(\phi)}$ is the selected $N_{T} \times N_{T}$ channel matrix. According to [22], $\beta_{k, j}^{(\phi)}$ is exponentially distributed and a upper bound for $\operatorname{Pr}\left(A_{i}\right)$ is given by [14] as

$$
\operatorname{Pr}\left(A_{i}\right) \leq\left[\operatorname{Pr}\left(\beta_{k_{1}, j_{1}}^{\left(\phi_{1}^{\prime}\right)} \leq x\right)\right]^{N_{R}-N_{T}+1} \doteq x^{N_{R}-N_{T}+1}, \forall i,
$$

then the diversity lower bound can be easily shown as

$$
d \geq N_{R}-N_{T}+1 .
$$

Combining (32) and (36), we finish the proof.

Remark 3: It is interesting to see that such a diversity order $d$ is the same as that of an $N_{T} \times N_{R}$ point-to-point MIMO system with ZF receiver [22] and an $N_{R} \times N_{T}$ point-to-point MIMO system with ZF transmitter [23] or TAS in conjunction with ZF receiver [14], [15] (as illustrated in Fig. 3 (a)). All of them have $N_{T}$ spatially-multiplexed streams while $N_{R}>N_{T} \geq 2$. This observation indicates that the proposed Max-Min optimization based RAS preserves the network diversity gain of the ZF based MIMO-PNC to be the same as the achievable link diversity gain of the component point-to-point MIMO link within the MIMO-TWRN.

Additionally, we also have a byproduct from the diversity analysis, i.e., the diversity order of the $K$-user MIMO-BC system (as illustrated in Fig. 3 (b) with $K \geq 2$ ) employing Max-Min optimization based TAS and ZF receivers. If we maintain the notation of the equivalent channel gains as $\left\{\beta_{k, j}^{(\phi)}\right\}, j=1,2, \ldots, N_{T}, k=1,2, \ldots, K$, then, we have the following corollary.

Corollary 1. For a $K$-user $(K \geq 1)$ MIMO-BC system with an $N_{R^{-}}$ antenna transmitter and $K N_{T}$-antenna ZF receivers, where $N_{T}$ data streams are broadcasted through $N_{T}$ out of $N_{R}$ transmit antenna, the Max-Min optimization based antenna selection,

$$
\text { AS IV : } \phi_{K-B C}^{*}=\arg \max _{\phi \in \Phi}\left(\beta_{m i n}^{(\phi)}\right),
$$

$\beta_{\text {min }}^{(\phi)}:=\min _{j \in\left\{1,2, \ldots, N_{T}\right\}, k \in\{1,2, \ldots, K\}}\left(\beta_{k, j}^{(\phi)}\right)$, achieves the diversity order

$$
d_{K-B C}=N_{R}-N_{T}+1 \text {. }
$$

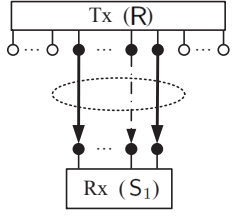

(a)

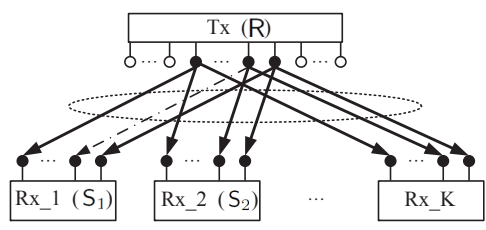

(b)
Fig. 3. (a) The MIMO point-to-point link for $\mathrm{R} \rightarrow \mathrm{S}_{1}$, wherein the dotdashed smaller arrow indicating the weakest link within the ellipse with equivalent channel gain $\beta_{1, \text { min }}^{(\phi)}$. (b) The $K$-user MIMO-BC, wherein the equivalent channel gain of the weakest link within the ellipse is $\beta_{\text {min }}^{(\phi)}$, also indicated by the dot-dashed smaller arrow.

TABLE I

THE ANTENNA SELECTION SCHEMES INVOLVED IN ALL THE TEST CASES

\begin{tabular}{|c|c|}
\hline AS & Description \\
\hline \hline AS I in (16), (20) & $\arg \max _{\phi \in \Phi}\left(\alpha_{\text {min }}^{(\phi)}\right)$ \\
\hline AS II in (17) & $\arg \max _{\phi^{\prime} \in \Phi} \min _{k \in\{1,2\}, j \in\left\{1, \ldots, N_{T}\right\}}\left(\beta_{k, j}^{\left(\phi^{\prime}\right)}\right)$ \\
\hline AS III in (44) & $\arg \max _{\phi \in \Phi} \min _{j \in\left\{1, \ldots, N_{T}\right\}}\left(\beta_{1, j}^{(\phi)}\right)$ \\
\hline AS IV in (37) & $\arg \max _{\phi \in \Phi} \min _{k \in\{1, \ldots, K\}, j \in\left\{1, \ldots, N_{T}\right\}}\left(\beta_{k, j}^{(\phi)}\right)$ \\
\hline
\end{tabular}

The proof of Corollary 1 can be done in a similar manner to that of Proposition 3, therefore we omit this proof for concision.

\section{Simulation And Discussion}

In this section, we provide simulations to evaluate the performance of the ZF based MIMO-PNC with R-RAS/C-RAS, and to validate the theoretical analysis of the end-to-end diversity order $d$. We consider the Rayleigh flat fading channels as assumed in Section II. For notational convenience, we use the number pair $\left(N_{R}, N_{T}\right)$ to represent the system configuration ${ }^{6}$ and $N_{T}$ also represents the spatialmultiplexed data schemes. In addition, we introduce the transmit bitSNR as SNR $:=E_{b} / N_{0}$ to evaluate system performance in all the following test cases, where $E_{b}=E_{S} / \log _{2} M$ is the average transmit bit power. More specifically, we assume that $E_{b}$ is normalized, i.e., $E_{b}=1$, thus the powers of the noise components at all three nodes are $N_{0}=\mathrm{SNR}^{-1}$ for all streams. For clear comparison, we also include the curves of $c \mathrm{SNR}^{-\alpha}$ as references, where $c$ is a casedependent coefficient and $\alpha$ is a positive integer. At the high SNR regime, if the bit or symbol error ratio (SER/BER) curve of one scheme tends to be parallel with $c \mathrm{SNR}^{-\alpha}$, we conclude that a diversity order of $\alpha$ is achieved. The BPSK modulation and the reciprocal channel are assumed for most of the test cases, if not otherwise specified. For $M$-QAM modulation, we only considered the rectangular constellation which can be decomposed into two orthogonal $\sqrt{M}$-ary PAM constellations. Additionally, Table I summarizes the antenna selection schemes involved in the following test cases.

TEST 1: Comparison between the proposed scheme and other counterparts. Fig. 4 provides the end-to-end average BER performance of different schemes to validate the advantages of the proposed ZF based MIMO-PNC scheme with C-RAS. It is shown that the BER performance of the original ZF based MIMO-PNC [6] with configuration $(2,2)$ is not better than the original SISO-PNC scheme [1] with configuration $(1,1)$ in fading channel, both showing a diversity order of $d=1$. For antenna configuration $(2,2)$, the ML

${ }^{6}$ For point-to-point MIMO or MIMO-BC system, $\left(N_{R}, N_{T}\right)$ represents $N_{R}$-antenna transmitter and $N_{T}$-antenna receiver(s). For MIMO-TWR, $\left(N_{R}\right.$, $N_{T}$ ) represents two $N_{T}$-antenna end nodes and on $N_{R}$-antenna relay. 


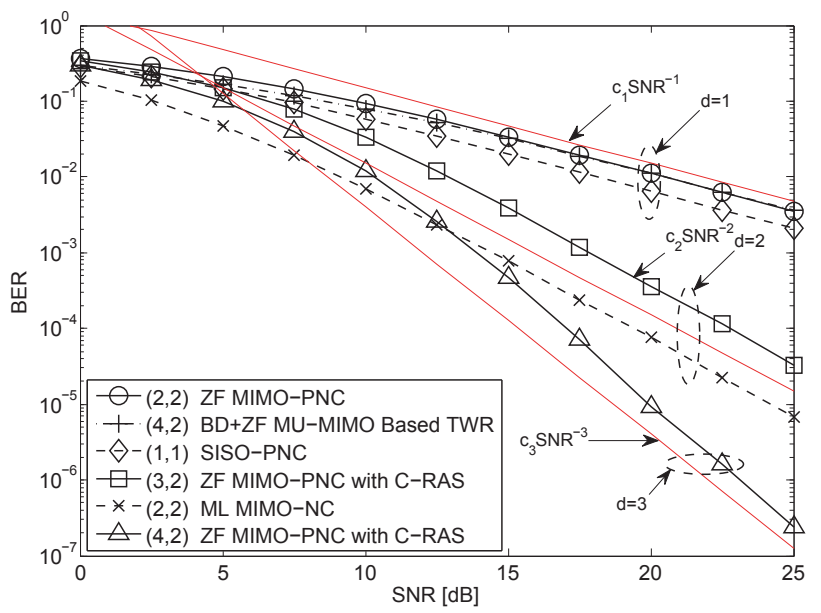

Fig. 4. The comparison of different two-way relay schemes regarding the average end-to-end BER performance with BPSK modulation.

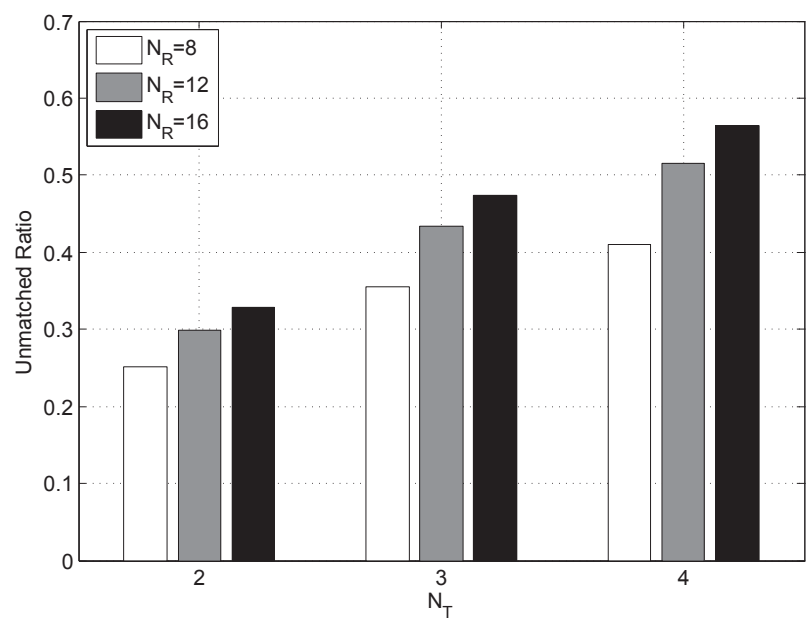

Fig. 5. Average unmatched ratio of the selected antenna subsets obtained from AS I and AS II with different $\left(N_{R}, N_{T}\right)$ pairs under the reciprocal channel condition.

based MIMO-NC ${ }^{7}$ shows superior performance with $d=2$ at the cost of the ML multi-user MIMO (MU-MIMO) detector at R and the ML MIMO detector at the two end nodes. The proposed scheme with configuration $(3,2)$ shows comparable performance as the ML based MIMO-NC with configuration $(2,2)$ in terms of diversity order. This observation indicates that the extra degree of freedom gained by deploying more antennas at $\mathrm{R}$ can bring selective diversity advantage to the simple ZF based MIMO-PNC with C-RAS. Therefore, the proposed scheme is easy to implement with diversity advantage. For antenna configuration $(4,2)$, the ZF based MU-MIMO transmission scheme is straightforwardly applicable to achieve the MIMO-TWR without NC/PNC, which exploits ZF detector in the MA phase and the block diagonalization (BD) based precoding [27] in the BC phase at the relay side. However, the ZF based MU-MIMO scheme wastes the potential diversity advantage offered by $R$, which only shows a diversity order of $d=1$. On the other hand, the proposed scheme shows superior performance with $(4,2)$, which provides a diversity order $d=3$.

TEST 2: Comparison between C-RAS and R-RAS. We com-

${ }^{7}$ MIMO-NC scheme explicitly decodes the individual symbols for both the end nodes in the MA phase; with the estimates of all the individual symbols, MIMO-NC generates the network-coded symbols by bit-wise XOR operation. This is in contract with the PNC which doesn't require explicitly decoding for individual symbols.

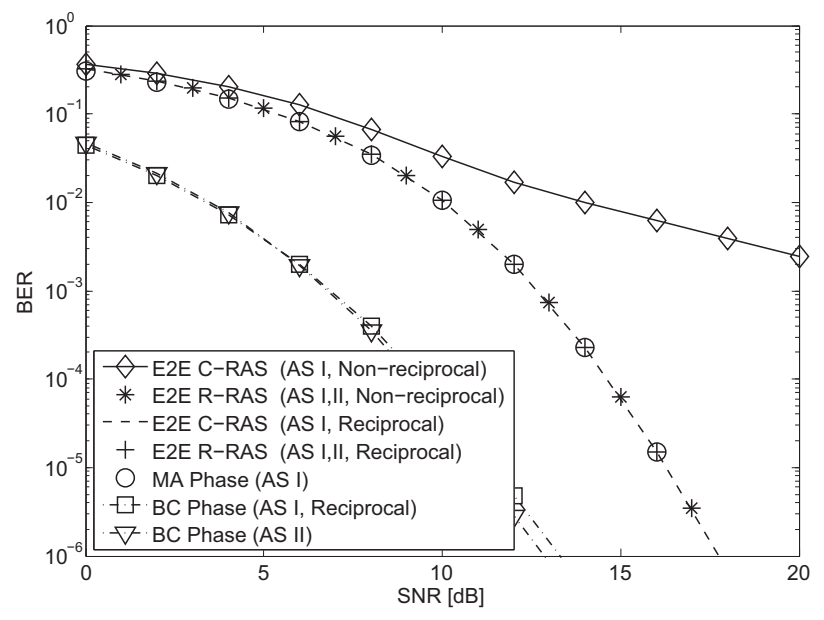

Fig. 6. Average end-to-end BER and phase BER performances of R-RAS and C-RAS with antenna configuration $(8,4)$ and BPSK modulation under reciprocal/non-reciprocal channel condition.

pare the C-RAS and R-RAS for ZF based MIMO-PNC in this test with both reciprocal and non-reciprocal channel conditions and various system antenna configurations of $\left(N_{R}, N_{T}\right)$, where $N_{R} \in$ $\{8,12,16\}$ and $N_{T} \in\{2,3,4\}$. Firstly, we give the unmatched ratio ${ }^{8}$ of the selected antenna subsets in MA phase and BC phase for the RRAS scheme under the condition of reciprocal channel. As illustrated in Fig. 5, for fixed $N_{R}\left(N_{T}\right)$ the unmatched ratio rises with $N_{T}$ $\left(N_{R}\right)$ and the unmatched ratio is relatively high in general. Note that C-RAS uses the same antenna subset for both transmission phases, the selected antenna subset is in fact only optimal for the MA phase transmission (cf. (20)). It seems reasonable to adopt R-RAS for better performance, considering that it optimizes both transmission phases. However, as shown in Fig. 6, for system with antenna configuration $(8,4)$ and antenna subsets unmatched ratio around $42 \%$, the end-toend BER performances of C-RAS and R-RAS are nearly overlapped with reciprocal channel, even though the link BER in the BC phase is indeed improved slightly by R-RAS in the high-SNR regime as compared to C-RAS. Therefore, the C-RAS is adequate for systems with reciprocal channel, where the overheads for CSI estimation involved in C-RAS as well as the computation cost for RAS are greatly reduced as compared with R-RAS. But R-RAS is still useful for systems operating with non-reciprocal channel. As shown in Fig. 6 , under non-reciprocal channel condition, R-RAS maintains its good performance as that with reciprocal channel while C-RAS shows poor performance. This observation indicates that two RAS operations are generally required to achieve a good performance when reciprocal channel is not assumed.

TEST 3: Validating the diversity analysis. Firstly, we compare the diversity orders achieved by different RAS approaches to validate some key equivalences that we have established during the diversity analysis. As shown in Fig. 7, all the antenna selection schemes exhibit the same diversity orders of $d=3$ with antenna configuration $(4,2)$ in different scenarios. In particular, the comparison between ZF based MIMO-TWR (PNC) with AS I ${ }^{9}$ (C-RAS) and two-user MIMO-BC with AS II validates the developing of equivalent relation in Proposition 2. This equivalent relation bridges the diversity analysis for MIMO-TWRC and MIMO-BC. Meanwhile, the comparison between the two-user MIMO-BC with AS II and the MIMO point-to-point system with AS III consolidates the relation in (32), which bridges the diversity analysis for MIMO-BC and MIMO point-to-point system. Moreover, we also show that the achieved diversity order with AS IV

\footnotetext{
${ }^{8}$ An unmatched event happens when $\phi_{R, M A}^{*} \neq \phi_{R, B C}^{*}$.
}

${ }^{9}$ The performances of ZF based MIMO-TWR with C-RAS and R-RAS are almost the same as shown in Fig. 6, we therefore omit the curves of R-RAS for clarity. 


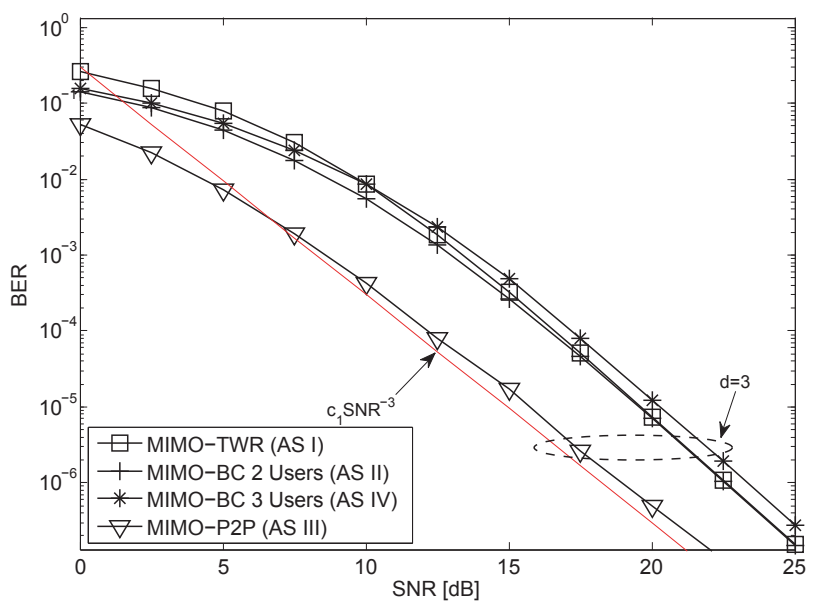

Fig. 7. Performance comparison of different antenna selection schemes for MIMO-TWR, MIMO-BC and MIMO point-to-point systems with antenna configuration $(4,2)$ and BPSK modulation.

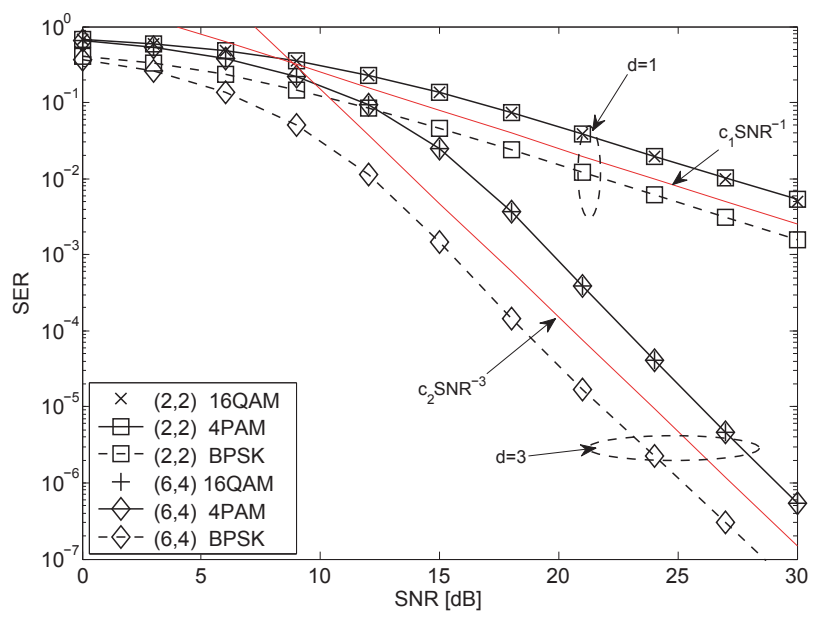

Fig. 8. The achieved diversity orders of the proposed scheme with different modulations including BPSK, 4PAM and 16QAM modulations, where the antenna configurations are $(2,2)$ and $(6,4)$.

for three-user MIMO-BC to validate Corollary 1. Next, we validate the diversity analysis for the proposed scheme with more antenna configurations and high-order modulations. As shown in Fig. 8, the proposed schemes with BPSK, QAM and 16-QAM all exhibit the same diversity order $d=1$ with antenna configuration $(2,2), d=3$ with $(6,4)$. Note that here the SER for 16-QAM is computed by the average SER for the corresponding 4-PAM signals on the quadrature carriers, therefore, the curves of 4-PAM and 16-QAM are almost overlapped.

\section{CONCLUSIONS}

We have investigated a spatial multiplexing oriented MIMOTWRN in which the relay node has $N_{R}$ antennas and each end node has $N_{T}$ antennas, where $N_{R}>N_{T} \geq 2$ is assumed. For this network, we have proposed a ZF based MIMO-PNC transmission scheme in conjunction with Max-Min optimization based RAS approaches, namely the R-RAS and its simplified version C-RAS. We have derived the explicit theoretical result that a diversity order of $d=N_{R}-N_{T}+1$ is attained by the proposed scheme with high-rate PNC employing $M$-PAM/QAM. We have also observed that with reduced implementation complexity the C-RAS provides almost the same end-to-end performance as the R-RAS under reciprocal channel condition.

\section{APPENDIX}

Lemma 1. : $d_{R}$ is limited by the weak phase diversity, i.e., $\min \left(d_{R, M A}, d_{R, B C}\right)$, where $d_{R, M A}$ and $d_{R, B C}$ can be defined with outage probability $\operatorname{Pr}\left(\alpha_{\min }^{\left(\phi_{R, M A}^{*}\right)} \leq x\right)$ and $\operatorname{Pr}\left(\beta_{\text {min }}^{\left(\phi_{R, B C}^{*}\right)} \leq x\right)$ respectively. In addition, $d_{C}$ can be defined with outage probability $\operatorname{Pr}\left(\alpha_{\min }^{\left(\phi_{R, M A}^{*}\right)} \leq x\right)$.

Proof: Let us first focus on $d_{R}$. According to the definition of $P_{E 2 E}^{\left(\phi, \phi^{\prime}\right)}$ in (9), the approximation of $P_{E 2 E, k, j}^{\left(\phi, \phi^{\prime}\right)}$ in (10) and the property of exponential equality [3], we have

$$
\bar{P}_{E 2 E}^{\left(\phi_{R, M A}^{*}, \phi_{R, B C}^{*}\right)}(\rho) \doteq \bar{P}_{M A}^{\left(\phi_{R, M A}^{*}\right)}(\rho)+\bar{P}_{B C, \max }^{\left(\phi_{R, B C}^{*}\right)}(\rho)
$$

where $\bar{P}_{M A}^{\left(\phi_{R, M A}^{*}\right)}(\rho):=\mathrm{E}_{H}\left\{P_{M A}^{\left(\phi_{R, M A}^{*}\right)}(\rho)\right\}, \bar{P}_{B C, \max }^{\left(\phi_{R, B C}^{*}\right)}(\rho):=$ $\mathrm{E}_{H}\left\{\max _{k, j}\left(P_{B C, k, j}^{\left(\phi_{R, B C}^{*}\right)}(\rho)\right)\right\}$. By substituting (38) into (21), we can redefine $d_{R}$ as

$$
\begin{aligned}
d_{R} & =-\lim _{\rho \rightarrow \infty}\left[\log \left(\bar{P}_{E 2 E}^{\left(\phi_{R, M A}^{*}\right)}(\rho)+\bar{P}_{B C, \text { max }}^{\left(\phi_{R, B C}^{*}\right)}(\rho)\right) / \log (\rho)\right] \\
& =\min \left(d_{R, M A}, d_{R, B C}\right),
\end{aligned}
$$

where and $d_{R, B C}=-\lim _{\rho \rightarrow \infty}\left[\bar{P}_{B C, \text { max }}^{\left(\phi_{R, B C}^{*}\right)}(\rho) / \log (\rho)\right]$ are the diversity orders of the MA and BC phases, respectively. By noting that $\left.P_{E 2 E}^{\left(\phi_{R}^{*}, M A\right.}\right)$ is bounded (cf. (11)) we have

$$
\begin{aligned}
\bar{P}_{M A}^{\left(\phi_{R, M A}^{*}\right)}(\rho) & \doteq \mathrm{E}_{H}\left\{\mathcal{Q}_{M A}^{\left(\phi_{R, M A}^{*}\right)}(\rho)\right\} \\
& \doteq \operatorname{Pr}\left(\log \left(1+\alpha_{\min }^{\left(\phi_{R}^{*}, M A\right.} \rho\right)<1\right)
\end{aligned}
$$

where $\mathrm{E}_{H}\left\{\mathcal{Q}_{M A}^{\left(\phi_{R, M A}^{*}\right)}(\rho)\right\}$ is interpreted as the average SEP of a point-to-point link and the second exponential equality follows [3], [14]. After some algebra, we come to the following exponential equation

$$
\bar{P}_{M A}^{\left(\phi_{R, M A}^{*}\right)}(\rho) \doteq \operatorname{Pr}\left(\alpha_{m i n}^{\left(\phi_{R, M A}^{*}\right)} \leq x\right),
$$

where $x=\rho^{-1}$, and we can redefine the diversity order $d_{R, M A}$ as (25). With the similar operations, we redefine $d_{R, B C}$ as (26).

Let us move on to the analysis for $d_{C}$ with reciprocal channel, i.e., $\mathbf{G}_{k}^{(\phi)}=\left(\mathbf{H}_{k}^{(\phi)}\right)^{T}, k=1,2$. For notational simplicity, we use a single superscript $\phi_{R, M A}^{*}$ to replace the antenna subset pair $\left(\phi_{R, M A}^{*}, \phi_{R, M A}^{*}\right)$. With similar treatment for (38), it is easy to see that

$$
\bar{P}_{E 2 E}^{\left(\phi_{R, M A}^{*}\right)}(\rho) \doteq \bar{P}_{E 2 E, \max }^{\left(\phi_{R, M A}^{*}\right)}(\rho),
$$

where $\quad \bar{P}_{E 2 E, \text { max }}^{\left(\phi_{R, M A}^{*}\right)}(\rho) \quad:=\quad \mathrm{E}_{H}\left\{P_{E 2 E, \text { max }}^{\left(\phi_{R, M A}^{*}\right)}(\rho)\right\} \quad$ and $P_{E 2 E, \text { max }}^{\left(\phi_{R, M A}^{*}\right)}(\rho):=\max _{k, j}\left(P_{E 2 E, k, j}^{\left(\phi_{R, M A}^{*}\right)}(\rho)\right)$. Besides, by noting the fact $\left.\alpha_{\min }^{\left(\phi_{R}^{*}, M A\right.}\right) \leq \beta_{k, j}^{\left(\phi_{R, M A}^{*}\right)}$ (cf. (19)), we can loose the bound for $P_{E 2 E, k, j}^{\left(\phi_{R, M A}^{*}\right)}$ in (13) and further give a bound of $P_{E 2 E, \max }^{\left(\phi_{R, M A}^{*}\right)}$ with reciprocal channel as

$$
\frac{2(M-1)}{M} \mathcal{Q}_{M A}^{\left(\phi_{R, M A}^{*}\right)} \leq P_{E 2 E, \max }^{\left(\phi_{R, M A}^{*}\right)} \leq \frac{4\left(M^{2}-1\right)}{M^{2}} \mathcal{Q}_{M A}^{\left(\phi_{R, M A}^{*}\right)},
$$

and, consequently, we have

$$
\bar{P}_{E 2 E, M a x}^{\left(\phi_{R, M A}^{*}\right)}(\rho) \doteq \mathrm{E}_{H}\left\{\mathcal{Q}_{M A}^{\left(\phi_{R, M A}^{*}\right)}(\rho)\right\} .
$$

Combining (42) and (43) we can redefine $d_{C}$ as (27) 
Lemma 2. : For an $N_{R} \times N_{T}$ MIMO point-to-point link with TAS and ZF receiver, the optimal achievable diversity order is obtained by the following Max-Min TAS approach

$$
\text { AS III : } \phi_{P 2 P}^{*}=\arg \max _{\phi \in \Phi}\left(\beta_{1, \text { min }}^{(\phi)}\right)
$$

where $\beta_{1, \text { min }}^{(\phi)}:=\min _{j}\left(\beta_{1, j}^{(\phi)}\right), j=1,2, \ldots, N_{T}$, and the optimal diversity order is given as

$$
d_{P 2 P}^{\left(\phi_{P 2 P}^{*}\right)}=\lim _{x \rightarrow 0} \log \left(\operatorname{Pr}\left(\beta_{1, m i n}^{\left(\phi_{P 2 P}^{*}\right)} \leq x\right)\right) / \log (x)=N_{R}-N_{T}+1 .
$$

Proof: See [14] and [15].

Lemma 3. There exists such a subset for $S_{i}, i \in\left\{1,2, \ldots, N_{u}\right\}$, which contains $N_{R}-N_{T}+1$ i.i.d. random variables as

$$
S_{i n d, i}=\left\{\beta_{k_{1}, j_{1}}^{\left(\phi_{1}^{\prime}\right)}, \beta_{k_{2}, j_{2}}^{\left(\phi_{2}^{\prime}\right)}, \ldots, \beta_{k_{N_{R}-N_{T}+1}, j_{N_{R}-N_{T}+1}}^{\left(\phi_{N_{R}-N_{T}+1}^{\prime}\right)}\right\} \subset S_{i},
$$

where $\left\{\phi_{m}^{\prime}\right\}_{m=1}^{N_{R}-N_{T}+1}$ is an ordered set containing $N_{R}-N_{T}+$ 1 different elements from $\Phi$ and $k_{m} \in\{1,2\}$ is the node index; $j_{m}=l_{i}(m)$ is the index of the picked relay antenna from $\phi_{m}^{\prime}$, and $\left\{j_{m}\right\}_{m=1}^{N_{R}-N_{T}+1}$ are required to be $N_{R}-N_{T}+1$ different integers picked from $\left\{1,2, \ldots, N_{R}\right\}$. Moreover, it is also required that the picked antenna $j_{m}$ belongs to $\phi_{m}^{\prime}$ but not to $\phi_{m^{\prime}}^{\prime}$ when $m^{\prime} \geq m$.

Proof: To facilitate this proof, we divide the set $\mathfrak{S}=\left\{S_{i}\right\}_{i=1}^{N_{u}}$ into three disjoint subsets as $\mathfrak{S}=\mathfrak{S}_{1} \cup \mathfrak{S}_{2} \cup \mathfrak{S}_{1 \& 2}$, where

$$
\begin{aligned}
\mathfrak{S}_{k} & =\left\{S_{i}: u_{i}(q)=k, q=1,2, \ldots, Q\right\}, k=1,2, \\
\mathfrak{S}_{1 \& 2} & =\mathfrak{S} /\left(\mathfrak{S}_{1} \cup \mathfrak{S}_{2}\right) .
\end{aligned}
$$

It is noted that Lemma 7 in [14] has given an constructive method to prove the case of $\mathfrak{S}_{1} \cup \mathfrak{S}_{2}$. More specifically, [14] has shown that there exists such a subset for arbitrary $S_{i} \in \mathfrak{S}_{k}, k=1,2$, which contains $N_{R}-N_{T}+1$ i.i.d. random variables as

$S_{i n d, i}=\left\{\beta_{k, j_{1}}^{\left(\phi_{1}^{\prime}\right)}, \beta_{k, j_{2}}^{\left(\phi_{2}^{\prime}\right)}, \ldots, \beta_{k, j_{N_{R}-N_{T}+1}}^{\left(\phi_{N_{R}-N_{T}+1}^{\prime}\right)}\right\} \subset S_{i} \in \mathfrak{S}_{k}, k=1,2$, wherein $\left\{\phi_{m}^{\prime}\right\}_{m=1}^{N_{R}-N_{T}+1}$ and $\left\{j_{m}\right\}_{m=1}^{N_{R}-N_{T}+1}$ are defined and constrained in the same way as Lemma 3. We then proceed to prove the $\mathfrak{S}_{1 \& 2}$ case where the node index $k_{m}$ is not fixed for all $m=$ $1,2, \ldots, M$. In fact, as long as $\left\{\phi_{m}^{\prime}\right\}_{m=1}^{N_{R}-N_{T}+1}$ and $\left\{j_{m}\right\}_{m=1}^{N_{R}-N_{T}+1}$ are determined in the same way as that for $\mathfrak{S}_{k}$, the explicit values of $\left\{k_{m}\right\}$ do not change the i.i.d. property among the random variables in $S_{i n d, i}$ for $\mathfrak{S}_{1 \& 2}$. Based on the above arguments, we complete the proof.

\section{REFERENCES}

[1] S. Zhang, S. Liew, and P. Lam, "Hot topic: physical-layer network coding," in Proc. 12th ACM MobiCom, Los Angeles, CA, USA, Sept. 2006, pp. 358-365.

[2] A. Paulraj, D. Gore, R. Nabar, and H. Bolcskei, "An overview of MIMO communications-a key to gigabit wireless," Proceedings of the IEEE, vol. 92, pp. 198-218, Feb. 2004

[3] L. Zheng and D. Tse, "Diversity and multiplexing: a fundamental tradeoff in multiple-antenna channels," IEEE Trans. Inf. Theory, vol. 49, pp. 1073-1096, May 2003.

[4] S. Zhang, S. C. Liew, and L. Lu, "Physical layer network coding schemes over finite and infinite fields," in Proc. IEEE GLOBECOM, New Orleans, LA, USA, Dec. 2008, pp. 3784-3789.

[5] S. Zhang, S. Liew, H. Wang, and X. Lin, "Capacity of two-way relay channel," Access Networks, vol. 37, pp. 219-231, 2010.

[6] H. J. Yang, K. Lee, and J. Chun, "Zero-forcing based two-phase relaying," in Proc. IEEE ICC, Glasgow, Scotland, June 2007, pp. 52245228.

[7] D. Park, "Phase alignment schemes in multiple antenna two-way relay channels," IEEE Commun. Lett., vol. 15, pp. 287-289, Mar. 2011.

[8] T. Yang, X. Yuan, L. Ping, I. B. Collings, and J. Yuan, "A new eigen-direction alignment algorithm for physical-layer network coding in MIMO two-way relay channels," in Proc. IEEE ISIT, Saint Petersburg, Russia, May 2011, pp. 2253-2257.
[9] H. J. Yang and J. Chun, "Generalized Schur decomposition-based twoway relaying for wireless MIMO systems," in Proc. IEEE GLOBECOM, New Orleans, LA, USA, Dec. 2008, pp. 1-6.

[10] H. J. Yang, J. Chun, and A. Paulraj, "Asymptotic capacity of the separated MIMO two-way relay channel with linear precoding," in Proc. 48th Annu. Allerton Conf., Monticello, IL, USA, Oct. 2010, pp. 86-93.

[11] A. Khina, Y. Kochman, and U. Erez, "Physical-layer MIMO relaying," in Proc. IEEE ISIT, Saint Petersburg, Russia, May 2011, pp. 2437-2441.

[12] G. Amarasuriya, C. Tellambura, and M. Ardakani, "Performance analysis of zero-forcing for two-way MIMO AF relay networks," IEEE Wireless Commun. Lett., vol. 1, pp. 53-56, Apr. 2012.

[13] R. Wang and M. Tao, "Joint source and relay precoding designs for MIMO two-way relaying based on MSE criterion," IEEE Trans. Signal Process., vol. 60, pp. 1352-1365, March 2012.

[14] H. Zhang, H. Dai, Q. Zhou, and B. Hughes, "On the diversity order of spatial multiplexing systems with transmit antenna selection: A geometrical approach," IEEE Trans. Inf. Theory, vol. 52, pp. 5297-5311, Dec. 2006.

[15] J. Jalden and B. Ottersten, "On the maximal diversity order of spatial multiplexing with transmit antenna selection," IEEE Trans. Inf. Theory, vol. 53, pp. 4273-4276, Nov. 2007.

[16] M. Eslamifar, C. Yuen, W. H. Chin, and Y. L. Guan, "Max-min antenna selection for bi-directional multi-antenna relaying," in Proc. 71st VTC Spring, Taipei, Taiwan, May 2010, pp. 1-5.

[17] Y. Li, R. Louie, and B. Vucetic, "Relay selection with network coding in two-way relay channels," IEEE Trans. Veh. Technol., vol. 59, pp. 4489-4499, Nov. 2010.

[18] Q. Zhou, Y. Li, F. Lau, and B. Vucetic, "Decode-and-forward two-way relaying with network coding and opportunistic relay selection," IEEE Trans. Commun., vol. 58, pp. 3070-3076, Nov. 2010.

[19] G. Amarasuriya, C. Tellambura, and M. Ardakani, "Two-way amplifyand-forward MIMO relay networks with antenna selection," in Proc. IEEE GLOBECOM, Houston, TX, USA, Dec. 2011, pp. 1 -5.

[20] R. W. Heath Jr., S. Sandhu, and A. Paulraj, "Antenna selection for spatial multiplexing systems with linear receivers," IEEE Commun. Lett., no. 4, pp. 142-144, Apr. 2001.

[21] A. Molisch and M. Win, "MIMO systems with antenna selection," IEEE Microwave Magazine, vol. 5, pp. 46-56, Mar 2004.

[22] D. Gore, R. W. Heath Jr., and A. Paulraj, "On performance of the zero forcing receiver in presence of transmit correlation," in Proc. IEEE ISIT, Palais de Beaulieu, Lausanne, Switzerland, 2002, p. 159.

[23] A. H. Mehana and A. Nosratinia, "Diversity of MIMO linear precoding," submitted to IEEE Trans. Inf. Theory, vol. abs/1202.4736, 2012. [Online]. Available: http://arxiv.org/pdf/1202.4736v2.pdf

[24] M. Ju and I.-M. Kim, "Error performance analysis of BPSK modulation in physical-layer network-coded bidirectional relay networks," IEEE Trans. Commun., vol. 58, pp. 2770-2775, Oct. 2010.

[25] J. Proakis and M. Salehi, Digital communications. McGraw-Hill, New York, 1995.

[26] A. Papoulis and S. Pillai, Probability, random variables, and stochastic processes. McGraw-Hill, 2002.

[27] K.-K. Wong, R. Murch, and K. Letaief, "A joint-channel diagonalization for multiuser MIMO antenna systems," IEEE Trans. Wireless Commun., vol. 2, pp. 773-786, July 2003.

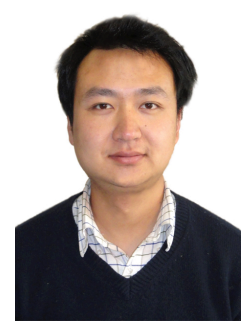

Hui Gao (S'10) received his B.S. degree in Information Engineering and Ph.D. degree in Signal and Information Processing from Beijing University of Posts and Telecommunications (BUPT), Beijing, China, in July 2007 and July 2012, respectively. From May 2009 to June 2012, he also served as a research assistant for the Wireless and Mobile Communications Technology R\&D Center, Tsinghua University, Beijing, China. From April 2012 to June 2012, he visited Singapore University of Technology and Design (SUTD), Singapore, as a research assistant. Since July 2012, he has been with SUTD as a Postdoc Researcher. His research interests include ultra-wideband wireless communications, physical-layer network coding and multiuser multiple-input-multipleoutput systems. 


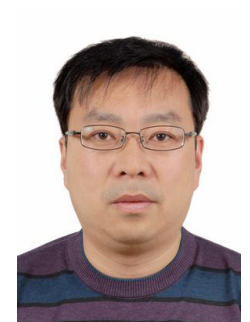

Tiejun Lv (M'08-SM'12) received the M.S. and $\mathrm{Ph} . \mathrm{D}$. degrees in electronic engineering from the University of Electronic Science and Technology of China, Chengdu, China, in 1997 and 2000, respectively. From January 2001 to December 2002, he was a Postdoctoral Fellow with Tsinghua University, Beijing, China. From September 2008 to March 2009, he was a Visiting Professor with the Department of Electrical Engineering, Stanford University, Stanford, CA. He is currently a Professor with the School of Information and Communication Engineering, Beijing University of Posts and Telecommunications. He is the author of more than 100 published technical papers on the physical layer of wireless mobile communications. His current research interests include signal processing, communications theory and networking. Dr. Lv is also a Senior Member of the Chinese Electronics Association. He was the recipient of the "Program for New Century Excellent Talents in University" Award from the Ministry of Education, China, in 2006.

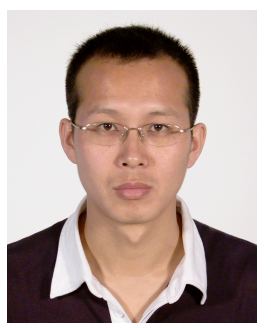

Shengli Zhang received his B. Eng. degree in electronic engineering and the M. Eng. degree in communication and information engineering from the University of Science and Technology of China (USTC), Hefei, China, in 2002 and 2005, respectively. He received the Ph.D. degree in the Department of Information Engineering, the Chinese University University of Hong Kong (CUHK), in 2008. From 2002 to 2005, he was with the Personal Communication Network and Spread Spectrum (PCNSS) Laboratory, USTC, as a Research Engineer involved in several National 863 Research Projects including the Beyond-3 Generation of Mobile System in China (FUTURE Plan). From 2002 to 2005, he was also a Research Engineer of the UTStarcom Wireless Soft Research Center, Hefei, China, involved in the research and implementation of the WCDMA communication systems. From Otc. 2008, he was a Research Associate in CUHK. Now, he is an associate professor with the Communication Engineering Department, Shenzhen University, China. His current research interests include wireless networks, wireless communication, physical layer network coding, and cooperative wireless networks.

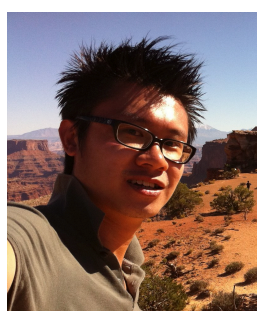

Chau Yuen (SM'12) received the B.Eng. and Ph.D. degrees from Nanyang Technological University, Singapore, in 2000 and 2004, respectively. He was a Postdoctoral Fellow with Lucent Technologies Bell Labs, Murray Hill, NJ, in 2005 and a Visiting Assistant Professor with Hong Kong Polytechnic University, Kowloon, Hong Kong, in 2008. During 2006-2010, he was with the Institute for Infocomm Research, A*STAR, Singapore, as a Senior Research Engineer. He joined Singapore University of Technology and Design, as an Assistant Professor in June 2010. He has published more than 100 research papers at international journals or conference proceedings. His current research interests include green communications, cognitive network, cooperative transmissions, network coding, and distributed storage. Dr. Yuen serves as an Associate Editor for the IEEE TRANSACTIONS ON VEHICULAR TECHNOLOGY.

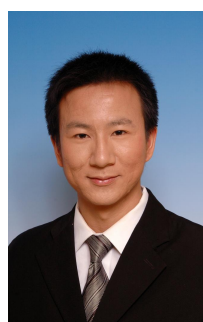

Shaoshi Yang (S'09) received the B.Eng. degree in information engineering from Beijing University of Posts and Telecommunications, Beijing, China, in 2006. He is currently working toward the Ph.D. degree in wireless communications with the School of Electronics and Computer Science, University of Southampton, Southampton, U.K., through scholarships from both the University of Southampton and the China Scholarship Council.

From November 2008 to February 2009, he was an Intern Research Fellow with the Communications Technology Laboratory, Intel Labs China, Beijing, where he focused on Channel Quality Indicator Channel design for mobile WiMAX (802.16 m). His research interests include multiuser dection/multiple-input-mutliple-output detection, multicell joint/distributed processing, green radio, and interference management.

Mr. Yang is a recipient of the PMC-Sierra Telecommunications Technology Scholarship, and a Junior Member of the Isaac Newton Institute for Mathematical Sciences, Cambridge, U.K., as well as a Technical Program Committee Member of the 23rd Annual IEEE International Symposium on Personal, Indoor, and Mobile Radio Communications (PIMRC 2012). 